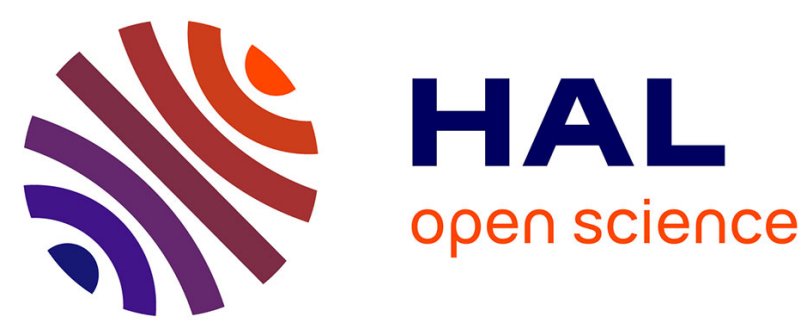

\title{
Convergence speed of nonlinear Luenberger observers
}

Vincent Andrieu

\section{To cite this version:}

Vincent Andrieu. Convergence speed of nonlinear Luenberger observers. SIAM Journal on Control and Optimization, 2014, 52 (5), pp.2831-2856. hal-01024164

\section{HAL Id: hal-01024164 \\ https://hal.science/hal-01024164}

Submitted on 15 Jul 2014

HAL is a multi-disciplinary open access archive for the deposit and dissemination of scientific research documents, whether they are published or not. The documents may come from teaching and research institutions in France or abroad, or from public or private research centers.
L'archive ouverte pluridisciplinaire HAL, est destinée au dépôt et à la diffusion de documents scientifiques de niveau recherche, publiés ou non, émanant des établissements d'enseignement et de recherche français ou étrangers, des laboratoires publics ou privés. 


\title{
CONVERGENCE SPEED OF NONLINEAR LUENBERGER OBSERVERS*
}

\author{
VINCENT ANDRIEU $\dagger$
}

\begin{abstract}
It is shown that under a weak observability assumption, the nonlinear Luenberger observer exists and ensures an exponential convergence rate of the estimate towards the state of the system. Moreover, it is shown that under the complete observability assumption, this class of observers is tunable. More specifically, it is shown that the estimate reaches a predetermined neighborhood of the state after a parameterizable time.
\end{abstract}

1. Introduction. State estimation is one of the major problems in control engineering. In the deterministic framework, an algorithm which can solve this problem is called a state observer. This algorithm is based on the knowledge of a dynamical model with measured outputs that accurately represents the considered physical phenomena and the sensors available. Since 1964 and the seminal work of Luenberger in [17], designing an observer for observable linear systems is now well understood. The approach of Luenberger can be decomposed into two steps. In the first one, a linear dynamic extension which defines a contraction, uniform in the measured output of the system, is introduced. In the second step, based on some observability properties, a linear map is obtained such that, when applied to the state of the dynamic extension, a state observer is obtained. With this approach, the estimate converges asymptotically toward the state of the system.

For nonlinear models, the problem is much more complicated and many different routes have been followed in order to extend this strategy. For instance we can refer to the popular linearization up to output injection (see for instance [14, 15, 25]). Few years back, Shoshitaishvili in [27. and more recently Kazantzis and Kravaris in [12] (see also [16]) have introduced a nonlinear local extension of the linear Luenberger observer. With their approach, it was shown that the existence of an observer around an equilibrium was obtained assuming local observability.

Recently, the non-local version of this tool has been studied in 13 and 3 . The interest of this approach lies in the fact that with a weak observability assumption (distinguishability of the state based on the past measured outputs), a nonlinear Luenberger observer exists and provides asymptotic state estimation of trajectories remaining in a given bounded set.

This observer approach has been employed with success for the design of an observer for permanent magnet synchronous motors with currents and voltages as only measurements (see [21]). The asynchronous motors has been studied in [1]. It has also been used to design an observer for harmonic oscillators in [23].

For linear systems, Luenberger observers have another fundamental property: their rate of convergence is exponential and configurable. Indeed, it is well known that when the system is linear and observable it is possible to choose the poles of the Luenberger observer. This property ensures an exponential decay of the euclidian norm of the estimation error with a term inside the exponential depending on the selected poles. In the non-linear context, this property is even more important. For instance, when we consider the stabilization by output feedback, it is essential to

*THE PRELIMINARY VERSION OF THIS WORK HAS BEEN PUBLISHED IN [2].

†Vincent Andrieu is with Université de Lyon, Lyon, F-69003, France, Université Lyon 1, CNRS, UMR 5007, LAGEP (Laboratoire d'Automatique et de GEnie des Procédés), 43 bd du 11 novembre, 69100 Villeurbanne, France http://sites.google.com/site/vincentandrieu/ 
ensure a specific convergence rate to get stability of the closed loop system (see [28] [10] or [4] for instance).

However, although the observer of [3] may ensure the asymptotic convergence of the estimate toward the state of the system, no characterization of the convergence speed is given. In this paper, with an extra observability assumption (the infinitesimal distinguishability property as introduced by Gauthier and Kupka in 9]) it is shown that the convergence speed of a nonlinear Luenberger observer is exponential and that the argument of the exponential decay can be selected arbitrary large. Moreover, in the complete observability context, it is shown that this type of observer may be tunable (notion introduced in [5]). More precisely, if a precision and a time are given, it is possible to configure the algorithm to ensure that after this time, the norm of the estimation error will be smaller than the given accuracy.

The paper is organized as follows. In Section 2.1, the nonlinear Luenberger observer is presented in its global version and one of the results obtained from [3] is given. The first result which establishes a sufficient condition to get exponential convergence of the nonlinear Luenberger observer is stated in Section 2.4. The main part of the proof of this result is given in Section 3. The complete observability context is studied in Section 4 . This section provides also a comparison between the two observability contexts. Section 5 contains all the technical proofs of the results stated all along the paper. Finally, Section 6 gives the conclusion.

\section{Notations}

- All along the paper, for all $x$ in $\mathbb{R}^{n}$ or $\mathbb{C}^{n},|x|$ denotes the norm 2 . In other word, we have $|x|=\sqrt{\langle\bar{x}, x\rangle}$. If $Q$ is a matrix in $\mathbb{C}^{n \times n},|Q|$ is the induced norm 2.

- For $Q$, a $m \times n$ complex matrix, $Q^{*}$ denotes its conjugate transpose $n \times m$ matrix.

- $I_{n}$ denotes the identity matrix in $\mathbb{R}^{n \times n}$.

\section{Exponential convergence of nonlinear Luenberger observers.}

2.1. Structure of a Nonlinear Luenberger observer. Consider a nonlinear system described by the following equation 1 .

$$
\dot{x}=f(x) \quad, \quad y=h(x),
$$

where $f: \mathbb{R}^{n} \rightarrow \mathbb{R}^{n}$ and $h: \mathbb{R}^{n} \rightarrow \mathbb{R}$ are two sufficiently smooth functions. For all $x$ in $\mathbb{R}^{n}$, the solution of System 2.1 initiated from $x$ at time 0 is denoted $X(x, t)$.

For all $x$ in a given open set $\mathcal{A}$ in $\mathbb{R}^{n}$, the maximal time interval of definition in $\mathcal{A}$ is denoted $\left(\sigma_{\mathcal{A}}^{-}(x), \sigma_{\mathcal{A}}^{+}(x)\right)$. More precisely, for all $x$ in $\mathcal{A}, X(x, t)$ is in $\mathcal{A}$ for all $t$ in $\left(\sigma_{\mathcal{A}}^{-}(x), \sigma_{\mathcal{A}}^{+}(x)\right)$. And if $X\left(x, \sigma_{\mathcal{A}}^{-}(x)\right)$ (respectively $\left.X\left(x, \sigma_{\mathcal{A}}^{+}(x)\right)\right)$ exists, then $X\left(x, \sigma_{\mathcal{A}}^{-}(x)\right) \notin \mathcal{A}\left(\operatorname{resp} . X\left(x, \sigma_{\mathcal{A}}^{+}(x)\right) \notin \mathcal{A}\right)$.

Following [27, 12, 13, 3] a nonlinear Luenberger observer is a dynamical system of the form:

$$
\dot{z}=A z+B_{1 m} y \quad, \quad \hat{x}=T^{*}(z)
$$

\footnotetext{
${ }^{1}$ In this paper, for the sake of clarity only mono output time invariant systems are considered. However, following [21] it is possible to extend all these results (part from Propositions 4.2 and Proposition 4.3 to time varying systems provided all Assumptions imposed are uniform in the time. The extension to the multi-output case can also be performed following [3] in which the state $z$ of the observer is now seen as a matrix.
} 
with state $z$ (a complex vector) in $\mathbb{C}^{m}, A$ is a diagonal Hurwitz matrix in $\mathbb{C}^{m \times m}, B_{1 m}$ in $\mathbb{R}^{m}$ is defined as

$$
B_{1 m}=(1, \ldots, 1)^{\prime}
$$

and $T^{*}: \mathbb{C}^{m} \rightarrow \mathbb{R}^{n}$ is a continuous functions.

Following $27,12,13,3$, the motivation for this structure is to design the mapping $T^{*}$ as the left inverse of a $C^{1}$ mapping $22: \mathcal{A} \rightarrow \mathbb{R}^{m}$ satisfying

$$
\frac{\partial T}{\partial x}(x) f(x)=A T(x)+B_{1 m} h(x) .
$$

Indeed, it is easy to show that if $T$ is a mapping solution to 2.4 it yields for all $x$ such that $\sigma_{\mathcal{A}}^{+}(x)=+\infty$

$$
\lim _{t \rightarrow+\infty}|Z(z, x, t)-T(X(x, t))|=0 .
$$

Designing the map $T^{*}$ to a uniformly continuous left inverse of the mapping gives the asymptotic estimation of the solution with our observer.

Note that in 3, the nonlinear Luenberger observer considered is slightly more general since the function $y \mapsto B_{1 m} y$ is a nonlinear function of the output. However, since in our analysis we consider bounded sets, we can restrict ourselves to this specific case.

2.2. Existence and construction of a nonlinear Luenberger observer. As shown in [3], one of the the main interests of this approach is that its existence is guaranteed under some weak observability assumption. Indeed, assume that the past output path $t \mapsto h(X(x, t))$ restricted to the time in which the trajectory remains in a certain set is injective in $x$. Then, it is sufficient to choose $m=n+1$ generic complex eigenvalues for $A$ to get the existence of the existence of a function $T^{*}$ making System 2.2 an observer. The specific observability condition made is :

Assumption $1\left(\left(\mathcal{O}, \delta_{d}\right)\right.$-Backward distinguishability Property). There exists a bounded open set $\mathcal{O}$ of $\mathbb{R}^{n}$ and a strictly positive real number $\delta_{d}$ such that, for each pair of distinct points $x_{1}$ and $x_{2}$ in $\mathcal{O}$, there exists a negative time $t$ in I $^{3}$ $\left(\max \left\{\sigma_{\mathcal{O}+\delta_{d}}^{-}\left(x_{1}\right), \sigma_{\mathcal{O}+\delta_{d}}^{-}\left(x_{2}\right)\right\}, 0\right]$ such that :

$$
h\left(X\left(x_{1}, t\right)\right) \neq h\left(X\left(x_{2}, t\right)\right) .
$$

This distinguishability assumption says that the present state $x$ can be distinguished from other states in an open set containing $\mathcal{O}$ by looking at the past output path restricted to the time in which the solution remains in $\mathcal{O}+\delta_{d}$.

One of the results obtained in $[3$ can be reformulated as follows.

Theorem 2.1 (3] Generic existence of Luenberger observer). Assume System 2.1) satisfies Assumption 1. Then, for all bounded open set $\mathcal{A}$ such that $\mathrm{Cl}(\mathcal{A}) \subset \mathcal{O}$,

\footnotetext{
${ }^{2}$ As shown in [3, we don't need $T$ to be $C^{1}$ as long as the Lie derivative of $T$ along $f$ exists.

${ }^{3}$ Given a subset $S \subseteq \mathbb{R}^{n}$ and a strictly positive real real number $\delta, \mathcal{S}+\delta$ is the open set defined as ,

$$
\mathcal{S}+\delta=\left\{x \in \mathbb{R}^{n}, \exists x_{\mathcal{S}} \in \mathcal{S},\left|x-x_{\mathcal{S}}\right|<\delta\right\}
$$


there exist a negative real number $\rho$ and zero Lebesgue measure subset $\mathcal{I}_{d}$ of $f^{4}\left(\mathbb{C}_{\rho}\right)^{n+1}$ such that for each $\left(\lambda_{1}, \ldots, \lambda_{n+1}\right)$ in $\left(\mathbb{C}_{\rho}\right)^{n+1} \backslash \mathcal{I}_{d}$ the following holds. There exists a continuous function $T^{*}$ and a continuous function $\beta$ which is decreasing in its second component such that for all $x$ in $\mathcal{A}$ and all $z$ in $\mathbb{C}^{n+1}$

$$
\lim _{t \rightarrow+\infty} \beta(z, t)=0
$$

and,

$$
|\hat{X}(x, z, t)-X(x, t)| \leq \beta(z, t), \forall t \in\left[0, \sigma_{\mathcal{A}}^{+}(x)\right),
$$

where,

$$
\hat{X}(x, z, t)=T^{*}(Z(x, z, t))
$$

and where $(Z(x, z, t), X(x, t))$ is the solution of System (2.1) and 2.2) with $A=$ $\operatorname{Diag}\left\{\lambda_{1}, \ldots, \lambda_{n+1}\right\}$.

In [3], this result was not stated in this way. However, it is a direct consequence of the fact that we restrict our analysis to a bounded set $\mathcal{A}$.

The $\beta$ function in the previous equation allows us to assess the quality of the estimate on the time of existence of the solution of the model in the set $\mathcal{A}$. As long as the solution remains in the set $\mathcal{A}$, the quality of the estimate increases due to the fact that this function is strictly decreasing with time. Moreover, if for a given initial condition $x$ in $\mathcal{A}$, the corresponding state trajectory $X(x, t)$ remains in $\mathcal{A}$ in forward time (i.e. $\sigma_{\mathcal{A}}^{+}(x)=+\infty$ ), the estimation asymptotically converges to the state. Note moreover that as shown in [3], the bound exhibits the distance between $z$ and $T(x)$. Hence, if $z$ was initiated at $T(x)$ then the estimation $\hat{x}(t)$ would follow the true state of the system.

It may be noticed that since we don't know the initial state of the system, we don't know in general $\sigma_{\mathcal{A}}^{+}(x)$. Consequently, we don't know the time length in which inequality (2.7) holds. However, in practice, we may have an a priori knowledge of a compact set of initial conditions $\mathcal{I}$ included in $\mathcal{A}$. Then, we know that inequality (2.7) holds at least in $\max _{x \in \mathcal{I}} \sigma^{+}(x)$. Note moreover, that the knowledge of the function $\beta$ and the value of the estimate implies that we know a ball in which the true state is. As long as this ball remains in $\mathcal{A}$, we know that the estimate is valid. From this, we get the following trivial corollary which gives a time length in which inequality 2.7) holds.

COROLlary 2.2. Assume all assumptions of Theorem 2.1 hold. Let $t_{0}=\max _{x \in \mathcal{I}} \sigma^{+}(x)$ and $z$ be in $\mathbb{C}^{n+1}$. Then inequality (2.7) holds for all $x$ in $\mathcal{I}$ and for all $t$ such that one of the two following items is satisfied.

- $t \leq t_{0}$;

- $t \geq t_{0}$ and for all $s$ in $\left[t_{0}, t\right] \mathcal{B}_{\hat{X}(x, z, t)}(\beta(z, s)) \subset \mathcal{A}$.

In order to give an explicit realization of the observer, we need first to find a solution to the partial differential equation (2.4). This equation is the corner stone of the nonlinear Luenberger observer methodology. Despite the fact that its existence is ensured with some weak assumptions, obtaining an explicit solution may be a hard

$$
\begin{aligned}
& { }^{4} \mathbb{C}_{\rho} \text { is the open subset of } \mathbb{C} \text { defined as } \\
& \qquad \mathbb{C}_{\rho}=\{\lambda \in \mathbb{C}: \operatorname{Re}(\lambda)<\rho\},
\end{aligned}
$$

where Re is the real part. 
task. Note however that on some examples this partial differential equation may be solved as [1, 21, 23]. Note moreover, that it is possible to perform a numerical approximation of this mapping. Indeed, it has been shown in [3] that an approximation of the mapping $T$ could be used provided the dynamics of the observer is modified. Following this remark, a numerical scheme has been introduced in 18 to construct a suitable approximation of the mapping $T$ given a generic nonlinear model.

The second step of the design is to compute $T^{*}$ the left inverse of the mapping $T$. And as in the high-gain methodology (see [9]), there is no general way of designing such mapping. Note that a possible expression can be given as the solution to a global optimization procedure :

$$
\hat{x}=T^{*}(z):=\operatorname{Argmin}_{x \in \mathrm{Cl}(\mathcal{A})}|z-T(x)|^{2} .
$$

In [18, some other numerical constructions of this left inverse have been given.

2.3. Example : The harmonic oscillator. As an illustrative example of the nonlinear Luenberger observer and following 23, consider the model of a harmonic oscillator with unknown angular velocity (see [6] for recent results on the same topic with some other approaches). This one is given as the following nonlinear model with state $x:=\left(x_{1}, x_{2}, x_{3}\right)$ satisfying

$$
\dot{x}_{1}=-x_{2}, \dot{x}_{2}=x_{3} x_{1}, \dot{x}_{3}=0, y=x_{1} .
$$

Given an initial condition $x_{a}:=\left(x_{a 1}, x_{a 2}, x_{a 3}\right)$ with $x_{a 3}>0$, the measured output is the time function defined for all positive timf $f^{5}$ as $y(t):=h\left(X\left(x_{a}, t\right)\right)=$ $x_{a 1} \cos \left(\sqrt{x_{a 3}} t\right)-\frac{x_{a 2}}{\sqrt{x_{a 3}}} \sin \left(\sqrt{x_{a 3}} t\right)$. It can be easily checked that the set $\mathcal{O}=\left\{x_{1}^{2}+\right.$ $\left.x_{2}^{2}>0, x_{3}>0\right\}$ is backward invariant. Moreover, consider two initial conditions denoted $\left(x_{a}, x_{b}\right)$ in the open set $\mathcal{O}$. If $h\left(X\left(x_{a}, t\right)\right)=h\left(X\left(x_{b}, t\right)\right)$ on a time interval then it yields that $x_{a}=x_{b}$. Thus Assumption 1 is satisfied for all positive real number $\delta_{d}$. Hence we know with Theorem 2.1 that there exists a nonlinear Luenberger observer for the harmonic oscillator provided the initial condition is in a given bounded open subset of $\mathcal{O}$.

For instance, following [23] (see also [1]) the set $\mathcal{A}$ is selected as $\mathcal{A}:=\left\{x_{3} x_{1}^{2}+x_{2}^{2}>\right.$ $\left.\epsilon_{1}, \epsilon_{2}>x_{3}>\epsilon_{3}\right\}$. This selection guarantees that the set $\mathcal{A}$ is forward invariant. An (asymptotic) observer for this system is given as,

$$
\hat{x}=T^{*}(z), z=\left[z_{1}, z_{2}, z_{3}, z_{4}\right]^{T}, \dot{z}_{i}=\lambda_{i} z_{i}+y, i=1, \ldots, 4,
$$

where $\lambda_{i}$ are four distinct negative real numbers and $T^{*}: \mathbb{R}^{4} \rightarrow \mathcal{A}$ is a left inverse of the mapping $T: \mathcal{A} \rightarrow \mathbb{R}^{4}$ defined as

$$
T(x)=\left[T_{1}(x), T_{2}(x), T_{3}(x), T_{4}(x)\right]^{T}, T_{i}(x)=\frac{-\lambda_{i} x_{1}+x_{2}}{\lambda_{i}^{2}+x_{3}} .
$$

In [23] a continuous left inverse of this mapping is explicitly given.

Despite the fact that a nonlinear Luenberger observer may ensure asymptotic estimation of the state of the system, nothing is said concerning its convergence speed. In the next section, a sufficient condition is given under which exponential convergence

\footnotetext{
${ }^{5}$ This time function can be written in the form $y(t)=\mathbb{A} \sin (\omega t+\Phi)$ with $\mathbb{A}$ the amplitude of the signal and $w$ and $\Phi$ respectively frequency and phase.
} 
of the estimation error towards the origin is obtained. In other words, the function $\beta$ given in Theorem 2.1 is given as

$$
\beta(z, t)=M(z) \exp (-c t),
$$

where $c$ is a positive real number and the function $M$ is a continuous given later on.

2.4. Context and first result. In this section, a sufficient condition guaranteeing exponential convergence of the observer (2.2) is given. More precisely, in this section, the following two observability assumptions are imposed on the system (2.1).

Assumption 2 (O-Backward distinguishability Property). There exists an open set $\mathcal{O}$ of $\mathbb{R}^{n}$ such that, for each pair of distinct points $x_{1}$ and $x_{2}$ in $\mathcal{O}$, there exists a negative time $t$ in $\left(\max \left\{\sigma_{\mathbb{R}^{n}}^{-}\left(x_{1}\right), \sigma_{\mathbb{R}^{n}}^{-}\left(x_{2}\right)\right\}, 0\right]$ such that :

$$
h\left(X\left(x_{1}, t\right)\right) \neq h\left(X\left(x_{2}, t\right)\right) .
$$

Note that this Assumption is weaker then Assumption 1 previously defined. Indeed, now, nothing is said on how the output distinguishes two given initial conditions. Moreover, the set $\mathcal{O}$ may not be bounded. Note however that by taking $\mathcal{O}$ bounded and $\delta_{d}=+\infty$, we recover Assumption 1 .

The second sufficient condition is an observability assumption which characterizes how a small change of the state modifies the backward output path on the time of existence of the solutions.

This assumption is related to the backward distinguishability of the following time varying linear system with output defined for all $x$ in $\mathbb{R}^{n}$ and $t$ in $\left(\sigma_{\mathbb{R}^{n}}^{-}(x), \sigma_{\mathbb{R}^{n}}^{+}(x)\right)$ as

$$
\dot{\zeta}=\frac{\partial f}{\partial x}(X(x, t)) \zeta, y_{z}=\frac{\partial h}{\partial x}(X(x, t)) \zeta,
$$

whose solutions initiated from $\zeta$ at $t=0$ for a given $x$ is written $\mathcal{X}(x, \zeta, t)$.

Assumption 3 ( $\mathcal{O}$-Backward Infinitesimal distinguishability property). Given an open set $\mathcal{O}$ of $\mathbb{R}^{n}$, for all $x$ in $\mathcal{O}$, for all $\zeta$ in $\mathbb{R}^{n} \backslash\{0\}$, there exists a negative time $t$ in $\left(\sigma_{\mathbb{R}^{n}}^{-}(x), 0\right]$ such that

$$
y_{z}(t):=\frac{\partial h}{\partial x}(X(x, t)) \mathcal{X}(\zeta, x, t) \neq 0
$$

The first result of the paper can now be stated.

THEOREM 2.3 (Exponential Luenberger observers). Let $\mathcal{O}$ be an open set of $\mathbb{R}^{n}$. Assume System (2.1) satisfies Assumptions 2 and 3 with the same observability set $\mathcal{O}$. Then for all bounded open set $\mathcal{A}$ of $\mathbb{R}^{n}$ such that $\mathrm{Cl}(\mathcal{A}) \subset \mathcal{O}$, there exist a negative real number $\rho$, a zero Lebesgue measure subset $\mathcal{I}_{e}$ of $\left(\mathbb{C}_{\rho}\right)^{n+1}$ such that for each $\left(\lambda_{1}, \ldots, \lambda_{n+1}\right)$ in $\left(\mathbb{C}_{\rho}\right)^{n+1} \backslash \mathcal{I}_{e}$ the following holds. There exists a continuous function $T^{*}: \mathbb{C}^{n+1} \rightarrow \mathbb{R}^{n}$ and a continuous function $M: \mathbb{C}^{n+1} \rightarrow \mathbb{R}_{+}$such that for all $(x, z)$ in $\mathcal{A} \times \mathbb{C}^{n+1}$

$$
\mid T^{*}\left(Z(x, z, t)-X(x, t) \mid \leq M(z) \exp \left(\max _{i}\left\{\operatorname{Re}\left(\lambda_{i}\right)\right\} t\right), \forall t \in\left[0, \sigma_{\mathcal{A}}^{+}(x)\right),\right.
$$

and where $(X(x, t), Z(x, z, t))$ is the solution of System (2.1) and (2.2) initiated from $(x, z)$ at $t=0$ with $A=\operatorname{Diag}\left\{\lambda_{1}, \ldots, \lambda_{n+1}\right\}$. 
2.5. Example (The harmonic oscillator, continued). Note that if we consider the model of the harmonic oscillator given in (2.8), the associated linear time varying system defined in 2.11) becomes simply,

$$
\dot{\zeta}=\left[\begin{array}{ccc}
0 & -1 & 0 \\
X_{3}(x, t) & 0 & X_{1}(x, t) \\
0 & 0 & 0
\end{array}\right] \zeta, y_{z}=\zeta_{1},
$$

where $X_{1}$ and $X_{3}$ are respectively the first and the third component of the state trajectory $X(x, t)$. Consider $x_{a}$ in the open set $\mathcal{A}$ defined in Section 2.3, and assume there exists $\zeta$ in $\mathbb{R}^{3}$ such that $y_{z}(t):=\mathcal{X}_{1}(\zeta, x, t)=0$ for all negative time. It yields, $\zeta_{1}=0$ and $\dot{\mathcal{X}}_{1}(\zeta, x, t)=-\mathcal{X}_{2}(\zeta, x, t)=0 \forall t \leq 0$. Hence, it yields $\zeta_{2}=0$ and $X_{1}(x, t) \zeta_{3}=0 \forall t \leq 0$. Note that $x$ being in $\mathcal{A}$, we know that there exists $t<0$ such that $X_{1}(x, t) \neq 0$. This implies that $\zeta_{3}=0$. Consequently, Assumption 3 is satisfied.

With Theorem 2.3, it yields the existence of an exponential Luenberger observer for the harmonic oscillator. Actually, on this particular example it can be shown that provided $\lambda_{1}, \lambda_{2}, \lambda_{3}$ and $\lambda_{4}$ are 4 different negative real numbers, the observer given in 2.9. has an exponential convergence rate.

3. Proof of Theorem 2.3. The proof of Theorem 2.3 is based on several propositions. All proofs are given in Section 5 . In this section, we only state each of these propositions to get the main ideas of the way an exponential nonlinear Luenberger observer can be obtained.

3.1. A constructive proposition. The following Propositions summarizes the exponential nonlinear Luenberger observer approach.

Proposition 3.1. Let $\mathcal{A}$ be a bounded open set of $\mathbb{R}^{n}$. If there exists a $C^{1}$ function $T: \mathbb{R}^{n} \rightarrow \mathbb{C}^{m}$ which satisfies the following two points:

1. $T$ is solution of the partial differential equation

$$
\frac{\partial T}{\partial x}(x) f(x)=A T(x)+B_{1 m} h(x), \forall x \in C l(\mathcal{A}),
$$

where $A=\operatorname{diag}\left\{\lambda_{1}, \ldots, \lambda_{m}\right\}$ is a Hurwitz $m \times m$ complex matrix and $B_{1 m}$ is defined in (2.3);

2. There exists a positive real number $L_{T}$ such that for all $\left(x_{1}, x_{2}\right)$ in $\operatorname{Cl}(\mathcal{A}) \times$ $\mathrm{Cl}(\mathcal{A})$, the following inequality holds.

$$
\left|x_{1}-x_{2}\right| \leq L_{T}\left|T\left(x_{1}\right)-T\left(x_{2}\right)\right| ;
$$

then there exists a continuous function $T^{*}: \mathbb{C}^{m} \rightarrow \mathbb{R}^{n}$ such that for all $(x, z)$ in $\mathcal{A} \times \mathbb{C}^{m}$

$\mid T^{*}\left(Z(x, z, t)-X(x, t)\left|\leq \sqrt{n} L_{T}\right| z-T(x) \mid \exp \left(\max _{i}\left\{\operatorname{Re}\left(\lambda_{i}\right)\right\} t\right), \forall t \in\left[0, \sigma_{\mathcal{A}}^{+}(x)\right)\right.$,

and where $(X(x, t), Z(x, z, t))$ is the solution of System (2.1) and 2.2) initiated from $(x, z)$ at $t=0$ with $A=\operatorname{Diag}\left\{\lambda_{1}, \ldots, \lambda_{n+1}\right\}$.

The proof of Proposition 3.1 can be found in the section 5.1.

With Proposition 3.1 it is established that an exponential nonlinear Luenberger observer is obtained provided we find a $C^{1}$ function $T$ solution of the partial differential equation (3.1) and which is uniformly injective in the sense of equation 3.2 . If we 
compare with [3, Theorem 2.10], in which only existence of a nonlinear Luenberger observer was obtained, inequality $(3.2)$ is replaced by the following inequality,

$$
\left|x_{1}-x_{2}\right| \leq \gamma\left(\left|T\left(x_{1}\right)-T\left(x_{2}\right)\right|\right) ;
$$

in which $\gamma$ is any class $\mathcal{K}_{\infty}$ function. If we compare inequalities $(3.4)$ and $(3.2)$, it is now required that we have a linear growth condition on the function $T$. This further requirement allow us to conclude on the exponential convergence of the obtained observer.

This further property may be obtained if the function $T$ is injective and its jacobian is full rank. This is stated in the following Lemma.

Lemma 3.2. Let $\mathcal{A}$ be a bounded open set of $\mathbb{R}^{n}$. If there exists a $C^{1}$ function $T: \mathbb{R}^{n} \rightarrow \mathbb{C}^{m}$ which satisfies the following two points:

1. The function $T$ is injective on $\operatorname{Cl}(\mathcal{A})$;

2. For all $x$ in $\operatorname{Cl}(\mathcal{A})$ the $m \times n$ complex matrix $\frac{\partial T}{\partial x}(x)$ is rank $n$; then there exists a positive real number $L_{T}$ such that (3.2) is satisfied.

The proof of Lemma 3.2 can be found in the section 5.2 .

With Proposition 3.1 and Lemma 3.2 it can be checked that to prove Theorem 2.3 it is required to find a solution to the partial differential equation (3.1) for all $x$ in $\mathrm{Cl}(\mathcal{A})$ such that this one is injective in $\mathrm{Cl}(\mathcal{A})$ and such that its gradient is full rank. In the remaining part of this Section, it is shown that this is indeed the case for almost all Hurwitz diagonal matrix $A$ provided we select $m=n+1$.

3.2. Solutions of the PDE given in (3.1). As proposed in [3] (see also [13]), given a bounded open set $\mathcal{A}$ and a diagonal Hurwitz matrix $A$ a solution of the partial differential equation (3.1) can be simply expressed as,

$$
T(x)=\int_{-\infty}^{0} \exp (-A s) B_{1 m} h(\breve{X}(x, s)) d s,
$$

where $\breve{X}: \mathbb{R}^{n} \times \mathbb{R} \rightarrow \mathbb{R}^{n}$ is the solution of the modified system

$$
\dot{x}=\breve{f}(x)=\chi(x) f(x),
$$

where $\chi: \mathbb{R}^{n} \rightarrow \mathbb{R}$ is a $C^{\infty}$ function such that

$$
\chi(x)= \begin{cases}0 & x \notin \mathcal{A}^{\prime}+\delta_{b} \\ 1 & x \in \mathcal{A}^{\prime}\end{cases}
$$

where $\delta_{b}$ is any positive real number and $\mathcal{A}^{\prime}$ is a bounded open set such that $\mathrm{Cl}(\mathcal{A}) \subset$ $\mathcal{A}^{\prime}$.

Proposition 3.3. Consider a bounded open set $\mathcal{A}$ and $A=\operatorname{diag}\left\{\lambda_{1}, \ldots, \lambda_{m}\right\}$ a Hurwitz $m \times m$ complex matrix. For all positive real number $\delta_{b}$ and open set $\mathcal{A}^{\prime}$ such that $\mathrm{Cl}(\mathcal{A}) \subset \mathcal{A}^{\prime}$ the function $T: \mathbb{R}^{n} \rightarrow \mathbb{C}^{m}$ given in 3.5) is a continuous function which satisfies (3.1). Moreover there exists a negative real number $\rho$ such that if $\max _{i=1, \ldots, n+1}\left\{\operatorname{Re}\left(\overline{\lambda_{i}}\right)\right\}<\rho$ then the function $T$ is $C^{2}$.

The proof of Proposition 3.3 can be found in the section 5.3 .

In the case where the set $\mathcal{A}$ is not bounded, the existence of a solution to a partial differential equation similar to (3.1) can still be obtained provided the linear mapping $B_{1 m}$ is replaced by a continuous function (see [3] for more details).

Moreover, when the set $\mathcal{A}$ is backward invariant, it can be shown that the restriction of the solution of $(3.1)$ to $\mathcal{A}$ is unique. 
In the following two subsections based on the two observability assumptions it is shown that if the set $\mathcal{A}^{\prime}$ is properly selected it yields that generically on the eigenvalues of the matrix $A$ this function is an injective immersion.

3.3. Selection of $\mathcal{A}^{\prime}$. As noticed in [13, the function $T$ can be seen as a projection of the function $t \in \mathbb{R}_{-} \mapsto h(\breve{X}(x, s))$ on a specific basis of function. We wish this function $T$ to inherit the injectivity property of the mappings $x \mapsto\left(t \in \mathbb{R}_{-} \mapsto\right.$ $h(\breve{X}(x, s)))$. However, if we which this mapping to be injective the set $\mathcal{A}^{\prime}$ needs to be properly defined. Roughly speaking, the set $\mathcal{A}^{\prime}$ should be selected to ensure that we don't loose any backward observability properties by restricting ourself to the function $x \mapsto\left(t \in \sigma_{\mathcal{A}^{\prime}}^{-} \mapsto h(X(x, s))\right)$. This is exactly what is done in the following proposition.

Proposition 3.4. Let $\mathcal{O}$ be an open set of $\mathbb{R}^{n}$. Assume System (2.1) satisfies Assumptions 2 and 3 with the same observability set $\mathcal{O}$. Then for all bounded open set $\mathcal{A}$ such that $\mathrm{Cl}(\mathcal{A}) \subset \mathcal{O}$ there exist two bounded open sets $\mathcal{A}^{\prime}$ and $\mathcal{A}^{\prime \prime}$ with $\mathrm{Cl}(\mathcal{A}) \subset \mathcal{A}^{\prime \prime}$ such that the following holds.

1. For each pair of distinct points $\left(x_{1}, x_{2}\right)$ in $\mathrm{Cl}\left(\mathcal{A}^{\prime \prime}\right)^{2}$, there exists a negative time $t$ in $\left(\max \left\{\sigma_{\mathcal{A}^{\prime}}^{-}\left(x_{1}\right), \sigma_{\mathcal{A}^{\prime}}^{-}\left(x_{2}\right)\right\}, 0\right]$ such that :

$$
h\left(X\left(x_{1}, t\right)\right) \neq h\left(X\left(x_{2}, t\right)\right) ;
$$

2. for all $x$ in $\operatorname{Cl}\left(\mathcal{A}^{\prime \prime}\right)$, for all $v$ in $\mathbb{R}^{n} \backslash\{0\}$, there exists a negative time $t$ in $\left(\sigma_{\mathcal{A}^{\prime}}^{-}(x), 0\right]$ such that

$$
\left.\frac{\partial h(X(x, t)}{\partial x}\right) v \neq 0
$$

The proof of Proposition 3.4 can be found in the section 5.4 .

3.4. Generic properties of the solution of the PDE given in (3.1). Based on the result of the paper [3], it can be shown that if the matrix $A$ is obtained by generically selecting $m$ eigenvalues, the function $T$, solution of the PDE (3.1) which is defined in (3.5) with $\mathcal{A}^{\prime}$ obtained from Proposition 3.4, is injective provided System 2.1. is backward distinguishable (i.e. Assumption 2 is satisfied). More precisely the result obtained in 3 is:

Proposition 3.5 (Generic Injectivity, [3]). Consider three bounded open sets $\mathcal{A}$, $\mathcal{A}^{\prime}$ and $\mathcal{A}^{\prime \prime}$ with $\mathrm{Cl}(\mathcal{A}) \subset \mathcal{A}^{\prime \prime}$ such that for each pair of distinct points $x_{1}$ and $x_{2}$ in $\left(\mathcal{A}^{\prime \prime}\right)^{2}$, there exists a negative time $t$ in $\left(\max \left\{\sigma_{\mathcal{A}^{\prime}}^{-}\left(x_{1}\right), \sigma_{\mathcal{A}^{\prime}}^{-}\left(x_{2}\right)\right\}, 0\right]$ such that :

$$
h\left(X\left(x_{1}, t\right)\right) \neq h\left(X\left(x_{2}, t\right)\right) .
$$

Then, there exist a negative real number $\rho$ and a subset $\mathcal{I} \subset\left(\mathbb{C}_{\rho}\right)^{n+1}$ of zero Lebesgue measure such that the function $T: \mathbb{R}^{n} \rightarrow \mathbb{C}^{n+1}$ defined by (3.5) from $\mathcal{A}^{\prime}$ is injective on $\mathrm{Cl}(\mathcal{A})$ provided $A$ is a diagonal Hurwitz matrix with $m=n+1$ complex eigenvalues $\lambda_{i}$ arbitrarily chosen in $\left(\mathbb{C}_{\rho}\right)^{n+1} \backslash \mathcal{I}$.

Consequently, to apply Proposition 3.1 it has to be shown that generically on $A$ and under Assumption 3, the function $T$ defined in 3.5 is such that for all $x$ in $\mathcal{O}$ the matrix $\frac{\partial T}{\partial x}(x)$ is full rank. This is proved by the following proposition.

Proposition 3.6 (Generically an immersion). Consider three bounded open sets $\mathcal{A}, \mathcal{A}^{\prime}$ and $\mathcal{A}^{\prime \prime}$ with $\mathrm{Cl}(\mathcal{A}) \subset \mathcal{A}^{\prime \prime}$ such that for all $x$ in $\mathrm{Cl}(\mathcal{A})$, for all $\zeta_{1}$ and $\zeta_{2}$ in $\mathbb{R}^{n}$, there exists a negative time $t$ in $\left(\sigma_{\mathcal{A}^{\prime}}^{-}(x), 0\right]$ such that

$$
\frac{\partial h}{\partial x}(X(x, t)) \mathcal{X}\left(\zeta_{1}, x, t\right) \neq \frac{\partial h}{\partial x}(X(x, t)) \mathcal{X}\left(\zeta_{2}, x, t\right) .
$$


Then, there exist a negative real number $\rho$ and a subset $\mathcal{I} \subset\left(\mathbb{C}_{\rho}\right)^{n+1}$ of zero Lebesgue measure such that for all $x$ in $\operatorname{Cl}(\mathcal{A})$, the $m \times n$ matrix $\frac{\partial T}{\partial x}(x)$ is full rank with $T$ defined by (3.5) from $\mathcal{A}^{\prime}$ provided $A$ is a diagonal Hurwitz matrix with $m=n+1$ complex eigenvalues $\lambda_{i}$ arbitrarily chosen in $\left(\mathbb{C}_{\rho}\right)^{n+1} \backslash \mathcal{I}$.

3.5. Proof of Theorem 2.3. Let $\mathcal{A}$ be a bounded open subset of $\mathbb{R}^{n}$ such that $\operatorname{Cl}(\mathcal{A}) \subset \mathcal{O}$. The system (2.1) satisfying Assumptions 2 and 3 it yields with Proposition 3.4 the existence of two bounded open sets $\mathcal{A}^{\prime}$ and $\mathcal{A}^{\prime \prime}$ with $\operatorname{Cl}(\mathcal{A}) \subset \mathcal{A}^{\prime \prime}$ such that Items 1 and 2 in Proposition 3.4 hold. With Proposition 3.3, 3.5 and 3.6 there exist a negative real number $\rho$, a subset $\mathcal{I} \subset \mathbb{C}^{n+1}$ of zero Lebesgue measure such that the function $T: \operatorname{Cl}(\mathcal{A}) \rightarrow \mathbb{C}^{(n+1)}$ defined by 3.5 is such that, provided $A$ is a diagonal matrix with $n+1$ complex eigenvalues $\lambda_{i}$ arbitrarily chosen in $\left(\mathbb{C}_{\rho}\right)^{n+1} \backslash \mathcal{I}$ the following holds.

1. For all $x$ in $\operatorname{Cl}(\mathcal{A}), T$ is a $C^{2}$ solution of the $\operatorname{PDE} 3.1$;

2. the function $T$ it is injective in $\operatorname{Cl}(\mathcal{A})$;

3. for all $x$ in $\operatorname{Cl}(\mathcal{A}), \frac{\partial T}{\partial x}(x)$ is full rank.

Consequently, given a matrix $A$ with eigenvalues in $\left(\mathbb{C}_{\rho}\right)^{n+1} \backslash \mathcal{I}$ and with Lemma 3.2 we can apply Proposition 3.1. This yields that the nonlinear Luenberger observer (2.2) satisfies the exponential convergence property 2.13 with $M$ defined as

$$
M(z)=\sqrt{n} L_{T}\left(|z|+\max _{x \in \operatorname{Cl}(\mathcal{A})}|T(x)|\right) .
$$

\section{Case of complete observability.}

4.1. The context. Another setup in which nonlinear Luenberger observers have been studied in [3] is when we have complete observability. This property is related to the mapping which to a point $x$ gives a finite number of successive Lie derivatives of the output. More precisely, for a given integer $m$, we introduce the function $H_{m}$ : $\mathbb{R}^{n} \rightarrow \mathbb{R}^{m}$

$$
H_{m}(x)=\left(\begin{array}{l}
h(x) \\
L_{f} h(x) \\
\vdots \\
L_{f}^{m-1} h(x)
\end{array}\right)
$$

Here, $L_{f}^{i} h$ denotes the $i$ th iterate Lie derivative, i.e. $L_{f}^{i+1} h=L_{f}\left(L_{f}^{i} h\right)$. Of course, for this to make sense, the functions $f$ and $h$ must be sufficiently smooth.

The complete observability context is described by the following two Assumptions.

Assumption 4 (Differential observability in $\mathcal{O}$ ). For all bounded open set $\mathcal{A}$ such that $\mathrm{Cl}(\mathcal{A}) \subset \mathcal{O}$ there exists a positive integer $m$ so that the function $H_{m}$ : $\mathbb{R}^{n} \rightarrow \mathbb{R}^{m}$ is injective when restricted to $\operatorname{Cl}(\mathcal{A})$.

Assumption 5 (Differential rank condition in $\mathcal{O}$ ). For all bounded open set $\mathcal{A}$ such that $\operatorname{Cl}(\mathcal{A}) \subset \mathcal{O}$ there exists a positive integer $m$ so that the function $H_{m}: \mathbb{R}^{n} \rightarrow \mathbb{R}^{m}$ is of rank $n$ in $\operatorname{Cl}(\mathcal{A})$. More precisely, for all $x$ in $\operatorname{Cl}(\mathcal{A})$, the matrix $\frac{\partial H_{m}}{\partial x}(x)$ is full rank.

With a Taylor expansion of the output path at $t=0$, we see that the injectivity of $H_{m}$ implies that the function which associates the initial condition $x$ to the output 
path, restricted to a very small time interval, is injective. This property is nicely exploited by observers with very fast dynamics as high gain observers (see [9).

Note moreover that it can be shown that this observer has an exponential convergence (see [9]). High-gain observers have an even stronger property in the sense that they are tunable as defined by G. Besançon in [5. More precisely they satisfy that if we know a bounded open set in which the unknown state is, a high gain observer can be tuned in order to guarantee an estimation error as small as wanted after any prescribed time. This is typically the kind of property needed in order to design a stabilizing output feedback following a seperation principle paradigm as in [28].

This tunable aspect can also be obtained for nonlinear Luenberger observers when considering the complete observability context. More precisely we get the following result.

TheOREm 4.1. Let $\mathcal{O}$ be an open subset of $\mathbb{R}^{n}$. Assume System (2.1) satisfies Assumptions 4 and $[5$ with the same observability set $\mathcal{O}$. Then for all bounded open set $\mathcal{A}$ such that $\operatorname{Cl}(\mathcal{A}) \subset \mathcal{O}$, there exists a positive integer $m$ such that for any diagonal Hurwitz complex $m \times m$ matrix $A$, for any positive real numbers $\left(t_{f}, \epsilon\right)$, there exists a real number $k^{*}$ such that, for any $k$ strictly larger than $k^{*}$, there exists a continuous function $T_{k}^{*}: \mathbb{C}^{m} \rightarrow \operatorname{Cl}(\mathcal{A})$ and a continuous function $M_{k}: \mathbb{C}^{m} \rightarrow \mathbb{R}_{+}$such that the nonlinear Luenberger observer

$$
\dot{z}=k A z+B_{1 m} y, \hat{x}=T_{k}^{*}(z)
$$

satisfies the following.

1. For all $(x, z)$ in $\mathcal{A} \times \mathbb{C}^{m}$

$$
\mid T_{k}^{*}\left(Z(x, z, t)-X(x, t) \mid \leq M_{k}(z) \exp \left(k \max _{i}\left\{\operatorname{Re}\left(\lambda_{i}\right)\right\} t\right), \forall t \in\left[0, \sigma_{\mathcal{A}}^{+}(x)\right),\right.
$$

and where $(X(x, t), Z(x, z, t))$ is the solution of System 2.1) and (4.2) with $A=\operatorname{Diag}\left\{\lambda_{1}, \ldots, \lambda_{m}\right\}$

2. For all $x$ in $\mathcal{A}$

$$
\mid X(x, t)-T_{k}^{*}\left(Z(x, 0, t) \mid \leq \epsilon, \forall t_{f} \leq t \leq \sigma_{\mathcal{A}}^{+}(x) .\right.
$$

The proof of Theorem 4.1 can be found in the section 5.8

4.2. Discussion on Assumptions. This setup has been popularized and studied in deep details by Gauthier and his coworkers (see [9] and the references therein, see also [24]). In particular, it is established in [8] that, for any generic pair $(f, h)$ in 2.1, it is sufficient to pick $m=2 n+1$ to obtain that the mapping $H_{m}$ is injective.

Moreover, in the analytic case, this set of Assumption is equivalent with Assumption 2 and 3 as shown by the following two propositions.

Proposition 4.2 ([11]). Assume the vector field $f$ and the function $h$ are both analytic with infinite convergence radius and satisfies the $\mathcal{O}$-backward distinguishability for a given open set $\mathcal{O}$ of $\mathbb{R}^{n}$ (i.e. Assumption 2 is satisfied). Then the system is also differentially observable in $\mathcal{O}$ (i.e. Assumption 4 is satisfied).

Proposition 4.3. Assume the vector field $f$ and the function $h$ are both analytic with infinite convergence radius and satisfies the $\mathcal{O}$-backward infinitesimal distinguishability property for a given open set $\mathcal{O}$ of $\mathbb{R}^{n}$ (i.e. Assumption 3 is satisfied). Then the system satisfies also the differential rank condition in $\mathcal{O}$ (i.e. Assumption 5 is satisfied).

The proofs of Proposition 4.2 and Proposition 4.3 are given respectively in Section 5.6 and 5.7 . 
4.3. Example (The harmonic oscillator, continued):. Consider again the model of the harmonic oscillator given in 2.8. This system satisfying assumptions 2 and 3 and being analytic, we know with propositions 4.2 and 4.3 that given a bounded set in $\mathcal{O}$ there exists $m$ such that the mapping $H_{m}(x)$ is injective and full rank (i.e. assumptions 4 and 5 are satisfied).

Actually, on this particular example it can be shown that the mapping $H_{4}: \mathcal{O} \rightarrow$ $\mathbb{R}^{4}$ given as:

$$
H_{4}(x)=\left[\begin{array}{c}
x_{1} \\
-x_{2} \\
-x_{3} x_{1} \\
x_{3} x_{2}
\end{array}\right]
$$

is injective in the set $\mathcal{O}$. Note moreover, that we have

$$
\frac{\partial H_{4}}{\partial x}(x)=\left[\begin{array}{ccc}
1 & 0 & 0 \\
0 & -1 & 0 \\
-x_{3} & 0 & -x_{1} \\
0 & x_{3} & x_{2}
\end{array}\right]
$$

which is full rank in $\mathcal{O}$. Consequently applying Theorem 4.1, it yields the existence of a tunable nonlinear Luenberger observer.

As illustration consider the Luenberger observer given in $(2.9)$ where $T^{*}$ is selected as

$$
\hat{x}=T^{*}(z)=\operatorname{Argmin}_{x \in \mathrm{Cl} \mathcal{A}} \sum_{j=1}^{4}\left(\lambda_{i} x_{1}+x_{2}-\left(\lambda_{i}^{2}+x_{3}\right) z_{i}\right)^{2}
$$

where $\mathcal{A}=\left\{x_{3} x_{1}^{2}+x_{2}^{2}>\epsilon_{1}, \epsilon_{2}>x_{3}>\epsilon_{3}\right\}$. For instance, a suitable numerical realization of the third component of this mapping is simply:

$$
\hat{x}_{3}=\max \left\{\min \left\{\left[\begin{array}{lll}
0 & 0 & 1
\end{array}\right]^{T} M(z)^{-1} N(z), \epsilon_{2}\right\}, \epsilon_{3}\right\},
$$

where

$$
M(z)=\left[\begin{array}{ccc}
\sum_{j=1}^{4} \lambda_{i}^{2} & \sum_{j=1}^{4} \lambda_{i} & -\sum_{j=1}^{4} \lambda_{i} z_{i} \\
\sum_{j=1}^{4} \lambda_{i} & 4 & -\sum_{j=1}^{4} z_{i} \\
-\sum_{j=1}^{4} \lambda_{i} z_{i} & -\sum_{j=1}^{4} z_{i} & \sum_{j=1}^{4} z_{i}^{2}
\end{array}\right], N(z)=\left[\begin{array}{c}
\sum_{j=1}^{4} \lambda_{i}^{3} z_{i} \\
\sum_{j=1}^{4} \lambda_{i}^{2} z_{i} \\
-\sum_{j=1}^{4} \lambda_{i}^{2} z_{i}^{2}
\end{array}\right] .
$$

Note that the saturation is employed to prevent the fact the matrix $M$ may be badly conditioned. Note however that another expression of $T^{*}$ well defined and continuous is given in 23. However, in the present context, there is no need of such use.

In Figure 4.1] is depicted the estimation of the frequency (i.e. $x_{3}$ ) when considering different values for the parameter $k$. Three scenarios have been considered $k=0.7, k=1, k=10$. As shown in Theorem 4.1. it can be seen that the convergence rate increases with $k$. The simulation has been performed in Matlab with an Euler integration for the observer and an Euler semi implicit integration for the harmonic oscillator. The integration stepsize has been tuned to $5 \cdot 10^{-4}$. The initial values of the model have been tuned to $x=[1,1,1]$. The parameters of the observer have been tuned to:

$$
\lambda_{1}=-0.7, \lambda_{1}=-0.9, \lambda_{1}=-1.1, \lambda_{1}=-1.3, \epsilon_{2}=0, \epsilon_{3}=5 .
$$




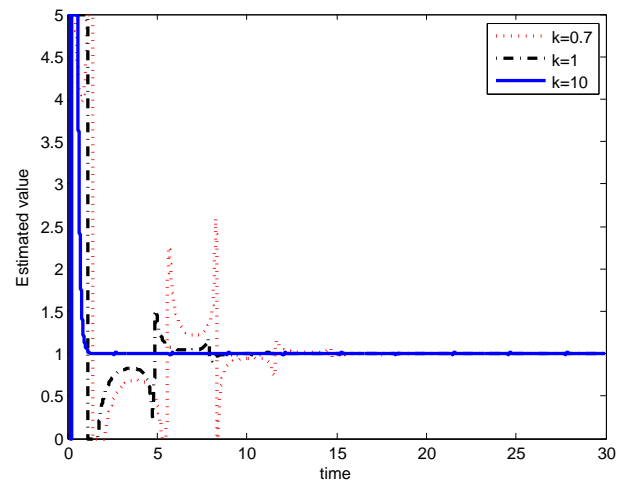

FIG. 4.1. Evolution with time of the estimation of $x_{3}$ for three different values of the parameter $k$.

\section{Proofs.}

5.1. Proof of Proposition 3.1. It is possible to define the function $T^{-1}$ : $T(\mathrm{Cl}(\mathcal{A})) \rightarrow \mathrm{Cl}(\mathcal{A})$ and this one satisfies,

$$
\left|T^{-1}\left(w_{1}\right)-T^{-1}\left(w_{2}\right)\right| \leq L_{T}\left|w_{1}-w_{2}\right|
$$

for all $\left(w_{1}, w_{2}\right)$ in $T(\mathrm{Cl}(\mathcal{A})) \times T(\mathrm{Cl}(\mathcal{A}))$. It yields that the function $T^{-1}: T(\mathrm{Cl}(\mathcal{A})) \rightarrow$ $\mathrm{Cl}(\mathcal{A})$ is globally Lipschitz. Hence, the function $T^{*}: \mathbb{C}^{m} \rightarrow \operatorname{Cl}(\mathcal{A})$ solution to our problem is a Lispchitz extension on the set $\mathbb{C}^{m}$ of this function. As exposed in 24] different solutions are possible. In this paper the authors suggest a constructive solution : the Mc-Shane formula (see [19] and more recently [18]). Following this 
approach we select $T^{*}=\left(T_{1}^{*}, \ldots, T_{n}^{*}\right)$ as the function defined by:

$$
T_{i}^{*}(w)=\inf _{z \in T(\mathrm{C}(\mathcal{A}))}\left\{\left(T^{-1}(z)\right)_{i}+L_{T}|z-w|\right\} .
$$

This function is such that for all $x$ in ${ }^{6} \operatorname{cl}(\mathcal{A})$,

$$
T^{*}(T(x))=x,
$$

and for all $(w, x)$ in $\mathbb{C}^{m} \times \operatorname{Cl}(\mathcal{A})$ it yields,

$$
\left|T^{*}(w)-x\right| \leq \sqrt{n} L_{T}|w-T(x)| .
$$

This implies that along the trajectories $(X(x, t), Z(x, z, t))$ of the system 2.1) and $(2.2)$ it yields for all $(x, z)$ in $\operatorname{Cl}(\mathcal{A}) \times \mathbb{C}^{m}$

$$
\left|T^{*}(Z(x, z, t))-X(x, t)\right| \leq \sqrt{n} L_{T}|Z(x, z, t)-T(X(x, t))|, \forall t \in\left[0, \sigma_{\mathcal{A}}^{+}(x)\right) .
$$

On another hand, the function $T$ is solution of the partial differential equation (3.1), consequently, this implies that along the trajectories of system (2.1) and (2.2), for all $(x, z)$ in $\operatorname{Cl}(\mathcal{A}) \times \mathbb{C}^{m}$

$$
Z(x, z, t)-T(X(x, t))=\exp (A t)(z-T(x)), \forall t \in\left[0, \sigma_{\mathcal{A}}^{+}(x)\right) .
$$

Note that since $A=\operatorname{Diag}\left(\lambda_{1}, \ldots, \lambda_{m}\right)$ with $\operatorname{Re}\left(\lambda_{i}\right)<0$, it yields that equation (3.3) holds and concludes the proof of Proposition 3.1 .

5.2. Proof of Lemma 3.2, Consider the function $\Delta: \mathrm{Cl}(\mathcal{A}) \times \mathrm{Cl}(\mathcal{A}) \rightarrow \mathbb{C}^{m}$ defined by,

$$
\Delta\left(x_{1}, x_{2}\right)=T\left(x_{1}\right)-T\left(x_{2}\right)-\frac{\partial T}{\partial x}\left(x_{2}\right)\left(x_{1}-x_{2}\right) .
$$

Note that the function $R: \mathrm{Cl}(\mathcal{A}) \rightarrow \mathbb{C}^{n \times m}$ given by,

$$
R(x)=\left(\left(\frac{\partial T}{\partial x}(x)\right)^{*} \frac{\partial T}{\partial x}(x)\right)^{-1}\left(\frac{\partial T}{\partial x}(x)\right)^{*},
$$

is well defined and continuous in $\operatorname{Cl}(\mathcal{A})$. For all $\left(x_{1}, x_{2}\right)$ in $\operatorname{Cl}(\mathcal{A}) \times \mathrm{Cl}(\mathcal{A})$, it yields 7 :

$$
\left|x_{1}-x_{2}\right| \leq R_{\max }\left(\left|T\left(x_{1}\right)-T\left(x_{2}\right)\right|+\left|\Delta\left(x_{1}, x_{2}\right)\right|\right),
$$

\footnotetext{
${ }^{6}$ Setting $z=T(x)$ in 5.2 , it yields

$$
T_{i}^{*}(T(x))-x_{i} \leq\left(T^{-1}(T(x))\right)_{i}-x_{i}=0
$$

Moreover, let $z_{0}=T\left(x_{0}\right)$ be such that $\inf _{z \in T(\operatorname{cr}(\mathcal{A}))}\left\{\left(T^{-1}(z)\right)_{i}+L_{T}|z-T(x)|\right\}=\left(T^{-1}\left(z_{0}\right)\right)_{i}+$ $L_{T}\left|z_{0}-T(x)\right|$, we have with 5.1)

$$
\begin{aligned}
T_{i}^{*}(T(x))-x_{i} & =\left(T^{-1}\left(T\left(x_{0}\right)\right)-T^{-1}(T(x))\right)_{i}+L_{T}\left|T\left(x_{0}\right)-T(x)\right| \\
& \geq L_{T}\left|T\left(x_{0}\right)-T(x)\right|-\left|\left(T^{-1}\left(T\left(x_{0}\right)\right)-T^{-1}(T(x))\right)_{i}\right| \\
& \geq 0
\end{aligned}
$$

${ }^{7}$ For a matrix $M$, the notation $|M|$ stands for the induced norm 2, i.e. $|M|=\|M\|_{2}$
} 
where $R_{\max }=\max _{x \in \mathrm{Cl}(\mathcal{A})}|R(x)|>0$. It yields, for all $\left(x_{1}, x_{2}\right)$ in $\mathrm{Cl}(\mathcal{A}) \times \mathrm{Cl}(\mathcal{A})$

$$
\left|x_{1}-x_{2}\right|\left(1-R_{\max } \frac{\left|\Delta\left(x_{1}, x_{2}\right)\right|}{\left|x_{1}-x_{2}\right|}\right) \leq R_{\max }\left|T\left(x_{1}\right)-T\left(x_{2}\right)\right| .
$$

Moreover, for all $a$ in $\mathrm{Cl}(\mathcal{A})$, there exists $\delta(a)>0$, such that, for all $x_{1}$ in $8 \mathcal{B}(a, \delta(a)) \cap$ $\mathrm{Cl}(\mathcal{A})$, it gives :

$$
\left|\Delta\left(x_{1}, a\right)\right| \leq \frac{1}{4 R_{\max }}\left|x_{1}-a\right|
$$

The function $\Delta$ being continuous in its second argument, for all $a$ in $\operatorname{Cl}(\mathcal{A})$, there exists a positive real number $\epsilon(a)$ such that, for all $\left(x_{1}, x_{2}\right)$ in $\mathcal{B}(a, \epsilon(a))^{2} \cap \mathrm{Cl}(\mathcal{A})^{2}$ :

$$
\left|\Delta\left(x_{1}, x_{2}\right)\right| \leq \frac{1}{2 R_{\max }}\left|x_{1}-x_{2}\right| .
$$

With 5.3$)$ it yields that for all $a$ in $\operatorname{Cl}(\mathcal{A})$,

$$
\left|x_{1}-x_{2}\right| \leq 2 R_{\max }\left|T\left(x_{1}\right)-T\left(x_{2}\right)\right|, \forall\left(x_{1}, x_{2}\right) \in \mathcal{B}(a, \epsilon(a))^{2} \cap \mathrm{Cl}(\mathcal{A})^{2} .
$$

On another hand, $\left\{\mathcal{B}\left(a, \frac{1}{2} \epsilon(a)\right), a \in \mathrm{Cl}(\mathcal{A})\right\}$ is a covering by open subset: $\mathrm{s}^{9}$ of the compact subset $\mathrm{Cl}(\mathcal{A})$. Hence, there exists $\left\{a_{1}, \ldots, a_{N}\right\}$ in $\mathrm{Cl}(\mathcal{A})^{N}$ with $N$ a positive integer, such that

$$
\mathrm{Cl}(\mathcal{A}) \subseteq \bigcup_{i=1, \ldots, N} \mathcal{B}\left(a_{i}, \frac{1}{2} \epsilon\left(a_{i}\right)\right)
$$

Since the function $T$ is injective on $\mathrm{Cl}(\mathcal{A})$, it is possible to define the positive real number :

$$
N_{\max }=\max _{\left(x_{1}, x_{2}\right) \in \Omega} \frac{\left|x_{1}-x_{2}\right|}{\left|T\left(x_{1}\right)-T\left(x_{2}\right)\right|}
$$

where $\Omega$ is the compact subset defined by,

$$
\Omega=\left\{\left(x_{1}, x_{2}\right) \in \operatorname{Cl}(\mathcal{A}) \times \operatorname{Cl}(\mathcal{A}):\left|x_{1}-x_{2}\right| \geq \epsilon_{\min }\right\},
$$

where,

$$
\epsilon_{\min }=\min _{i<N} \frac{1}{2} \epsilon\left(a_{i}\right)
$$

Consider now $\left(x_{1}, x_{2}\right)$ in $\mathrm{Cl}(\mathcal{A}) \times \mathrm{Cl}(\mathcal{A})$. Two cases can be distinguished:

1. $\left|x_{1}-x_{2}\right| \leq \epsilon_{\min }$ : since there exists $i<N$ such that $x_{2} \in \mathcal{B}\left(a_{i}, \frac{1}{2} \epsilon\left(a_{i}\right)\right)$, it yields,

$$
\begin{aligned}
\left|x_{1}-a_{i}\right| & \leq\left|x_{1}-x_{2}\right|+\left|x_{2}-a_{i}\right| \\
& \leq \epsilon_{\min }+\frac{1}{2} \epsilon\left(a_{i}\right) \\
& \leq \epsilon\left(a_{i}\right) .
\end{aligned}
$$

Hence, $x_{1} \in \mathcal{B}\left(a_{i}, \epsilon\left(a_{i}\right)\right)$, and consequently:

$$
\left|x_{1}-x_{2}\right| \leq 2 R_{\max }\left|T\left(x_{1}\right)-T\left(x_{2}\right)\right| .
$$

\footnotetext{
${ }^{8} \mathcal{B}\left(x_{c}, r\right)$ denotes the subset of $\mathbb{R}^{n}:\left\{x \in \mathbb{R}^{n},\left|x-x_{c}\right| \leq r\right\}$

${ }^{9}$ Depending on the shape of the set $\mathcal{A}$ it is possible to avoid the use of a covering to get the result.
} 
2. $\left|x_{1}-x_{2}\right| \geq \epsilon_{\min }$ : In this case $\left(x_{1}, x_{2}\right)$ is in $\Omega$ and consequently :

$$
\left|x_{1}-x_{2}\right| \leq N_{\max }\left|T\left(x_{1}\right)-T\left(x_{2}\right)\right| .
$$

Consequently, it yields that for all $\left(x_{1}, x_{2}\right)$ in $\operatorname{Cl}(\mathcal{A}) \times \operatorname{Cl}(\mathcal{A})$ :

$$
\left|x_{1}-x_{2}\right| \leq L_{T}\left|T\left(x_{1}\right)-T\left(x_{2}\right)\right|
$$

with, $L_{T}=\max \left\{N_{\max }, 2 R_{\max }\right\}$.

5.3. Proof of Proposition 3.3. First of all note that the function $\chi$ being $C^{\infty}$, the vector field $x \mapsto \chi(x) f(x)$ is as smooth as $f$. Hence, for all $x$ in $\mathbb{R}^{n}$ the function $\breve{X}(x, t)$, solution of system (3.6), are properly defined, unique, complete in positive and negative time and as smooth as $f$ in their $x$ component.

With the fact that $\mathcal{A}^{\prime}+\delta_{b}$ is bounded and backward invariant for the modified system [3.6), it yields that for all $i$ in $\{1, \ldots, m\}$ and all $(x, t)$ in $\mathcal{A}^{\prime}+\delta_{b} \times(-\infty, 0]$,

$$
\left|\exp \left(-\lambda_{i} t\right) h(\breve{X}(x, t))\right| \leq \exp \left(\left[-\operatorname{Re}\left(\lambda_{i}\right)\right] t\right) h_{\max },
$$

where $h_{\max }$ is the positive real number defined as,

$$
h_{\max }=\max _{x \in \mathrm{Cl}\left(\mathcal{A}^{\prime}+\delta_{b}\right)}|h(x)| .
$$

By Lebesgue dominated convergence Theorem it yields that, for all $x$ in $\mathcal{A}^{\prime}+\delta_{b}$, the function $T$ given in 3.5 defines a continuous function in $\mathrm{Cl}\left(\mathcal{A}^{\prime}+\delta_{b}\right)$. Moreover, for all $i$ in $\{1, \ldots, m\}$ and all $(x, t)$ in $\mathbb{R}^{n} \backslash \mathcal{A}^{\prime}+\delta_{b} \times(-\infty, 0]$,

$$
h(\breve{X}(x, t))=h(x),
$$

and consequently we see that the function $T$ given in 3.5 defines a continuous function in $\mathbb{R}^{n}$.

Then, for each $x$ in $\mathbb{R}^{n}$ and for each positive time $t$, we get :

$$
\begin{aligned}
T(\breve{X}(x, t))-T(x) & =\int_{-\infty}^{0} \exp (-A s) B_{1 m} h(\breve{X}(\breve{X}(x, t), s)) d s-T(x) \\
& =\exp (A t) \int_{-\infty}^{t} \exp (-A u) B_{1 m} h(\breve{X}(x, u)) d u-T(x) \\
& =(\exp (A t)-I) T(x)+\exp (A t) \int_{0}^{t} \exp (-A u) B_{1 m} h(\breve{X}(x, u)) d u
\end{aligned}
$$

Thus, we obtain, for all $x$ in $\mathbb{R}^{n}$,

$$
\chi(x) L_{f} T(x)=L_{\chi f} T(x)=\lim _{t \rightarrow 0} \frac{T(\breve{X}(x, t))-T(x)}{t}=A T(x)+B_{1 m} h(x) .
$$

With (3.7), this implies that (3.1) is satisfied.

Now following [22, Theorem 2.50], we show that by taking $\operatorname{Re}\left(\lambda_{i}\right)$ for all $i$ sufficiently negative, the function $T$ defined in 3.5 is $C^{2}$. First of all, for all $x$ in $\mathcal{A}^{\prime}+\delta_{b}$ and all $s$ in $\mathbb{R}_{-}$we have

$$
\frac{\partial^{2} \breve{X}}{\partial x \partial s}(x, s)=\frac{\partial \breve{f}}{\partial x}(\breve{X}(x, s)) \frac{\partial \breve{X}}{\partial x}(x, s)
$$


where $\breve{f}(x)=\chi(x) f(x)$. We can introduce the function $U$ defined as

$$
U(x, s)=\operatorname{trace}\left(\left(\frac{\partial \breve{X}}{\partial x}(x, s)\right)^{\prime} \frac{\partial \breve{X}}{\partial x}(x, s)\right) .
$$

Note that we have $U(x, 0)=n$. Moreover for all $x$ in $\mathcal{A}^{\prime}+\delta_{b}$ and for all $s$ in $\mathbb{R}_{-}$,

$$
U(x, s) \geq\left|\frac{\partial \breve{X}}{\partial x}(x, s)\right|^{2} .
$$

Also, it satisfies for all $s$ in $\mathbb{R}_{-}$

$$
\frac{\partial U}{\partial s}(x, s)=\operatorname{trace}\left(\left(\frac{\partial \breve{X}}{\partial x}(x, s)\right)^{\prime}\left[\left(\frac{\partial \breve{f}}{\partial x}(\breve{X}(x, s))\right)^{\prime}+\frac{\partial \breve{f}}{\partial x}(\breve{X}(x, s))\right] \frac{\partial \breve{X}}{\partial x}(x, s)\right) \text {. }
$$

Hence, employing the fact that for all $x$ in $\mathcal{A}^{\prime}+\delta_{b}$ the trajectories $s \mapsto \breve{X}(x, s)$ are bounded it gives the existence of a negative real number $\rho_{1}$ such that for all $x$ in $\mathcal{A}^{\prime}+\delta_{b}$ and for all $s$ in $\mathbb{R}_{-}$,

$$
\lambda_{\max }\left\{\left(\frac{\partial \breve{f}}{\partial x}(\breve{X}(x, s))\right)^{\prime}+\frac{\partial \breve{f}}{\partial x}(\breve{X}(x, s))\right\} \leq-2 \rho_{1} .
$$

Hence, it yields for all $x$ in $\mathcal{A}^{\prime}+\delta_{b}$ and for all $s$ in $\mathbb{R}_{-}$,

$$
\frac{\partial U}{\partial s}(x, s) \leq-2 \rho_{1} U(x, s) .
$$

Consequently, we obtain for all $x$ in $\operatorname{Cl}(\mathcal{A})+\delta_{b}$ and for all $s$ in $\mathbb{R}_{-}$,

$$
\left|\frac{\partial \breve{X}}{\partial x}(x, s)\right| \leq \sqrt{n} \exp \left(\rho_{1} s\right) .
$$

Hence, employing the fact that the bounded set $\mathcal{A}^{\prime}+\delta_{b}$ is backward invariant along the trajectories of the system $(3.6)$ we get for all $x$ in $\mathbb{R}^{n}$ and $s$ in $\mathbb{R}_{-}$,

$$
\left|\exp \left(-\lambda_{i} s\right) \frac{\partial h}{\partial x}(\breve{X}(x, s)) \frac{\partial \breve{X}}{\partial x}(x, s)\right| \leq \max \left\{d h_{\max },\left|\frac{\partial h}{\partial x}(x)\right|\right\} \sqrt{n} \exp \left(\left[\rho_{1}-\operatorname{Re}\left(\lambda_{i}\right)\right] s\right),
$$

where $d h_{\max }$ is the positive real number defined as,

$$
d h_{\max }=\max _{x \in \mathrm{Cl}\left(\mathcal{A}^{\prime}+\delta_{b}\right)}\left|\frac{\partial h}{\partial x}(x)\right| .
$$

With Lebesgue dominate convergence theorem, it can be established that the function

$$
\frac{\partial T}{\partial x}(x)=\int_{-\infty}^{0} \exp (-A s) \frac{\partial h}{\partial x}(\breve{X}(x, s)) \frac{\partial \breve{X}}{\partial x}(x, s) d s,
$$

is continuous and properly defined provided $\operatorname{Re}(\lambda)<\rho_{1}$ and consequently the function $T$ is $C^{1}$. Similarly, it can be shown that this function is $C^{2}$ provided $\operatorname{Re}(\lambda)<\rho_{e l}$ where $\rho_{e l}$ is a negative real number. 
5.4. Proof of Proposition 3.4 Let $\mathcal{A}^{\prime \prime}$ be any bounded open set such that

$$
\mathrm{Cl}(\mathcal{A}) \subset \mathcal{A}^{\prime \prime}, \operatorname{Cl}\left(\mathcal{A}^{\prime \prime}\right) \subset \mathcal{O} .
$$

Let $a$ be in $\operatorname{Cl}\left(\mathcal{A}^{\prime \prime}\right)$ and $b$ in ${ }^{10} \mathcal{S}_{n}$. Note that we have for all $t$ in $\left(\sigma_{\mathbb{R}^{n}}^{-}(a), \sigma_{\mathbb{R}^{n}}^{+}(a)\right)$,

$$
\frac{\partial^{2} X}{\partial t \partial x}(a, t) b=\frac{\partial f}{\partial x}(X(a, t)) \frac{\partial X}{\partial x}(a, t) b, \frac{\partial X}{\partial x}(a, 0) b=b .
$$

Consequently, the mapping $\left(X(a, t), \frac{\partial X}{\partial x}(a, t) b\right)$ defined on $\left(\sigma_{\mathbb{R}^{n}}^{-}(a), \sigma_{\mathbb{R}^{n}}^{+}(a)\right)$ is a solution of system 2.1) and 2.11) initiated from $(a, b)$. This system being locally Lipschitz, its trajectories are uniquely defined. Consequently for all $t$ in $\left(\sigma_{\mathbb{R}^{n}}^{-}(a), \sigma_{\mathbb{R}^{n}}^{+}(a)\right)$ we have,

$$
\mathcal{X}(b, a, t)=\frac{\partial X}{\partial x}(a, t) b .
$$

Since $a \in \operatorname{Cl}\left(\mathcal{A}^{\prime \prime}\right) \subset \mathcal{O}$, with Assumption 3 (setting $\zeta_{1}=b, \zeta_{2}=0$ ), there exists $t_{a, b}$ in $\left(\sigma_{\mathbb{R}^{n}}^{-}(a), 0\right]$ such that

$$
\left|\frac{\partial h}{\partial x}\left(X\left(a, t_{a, b}\right)\right) \frac{\partial X}{\partial x}\left(a, t_{a, b}\right) b\right|:=r(a, x)>0 .
$$

Consider now the function $\Delta(a, b, \ell)$ where $\ell$ is a positive real number defined when it exists by,

$$
\Delta(a, b, \ell)=h\left(X\left(a, t_{a, b}\right)\right)-h\left(X\left(a+\ell b, t_{a, b}\right)\right)-\ell \frac{\partial h}{\partial x}\left(X\left(a, t_{a, b}\right)\right) \frac{\partial X}{\partial x}\left(a, t_{a, b}\right) b .
$$

Note that the function $x \mapsto h(X(x, t))$ being $C^{1}$ we have,

$$
\lim _{\ell \rightarrow 0} \frac{\Delta(a, b, \ell)}{\ell}=0 .
$$

Hence, there exists $\ell_{0}$ such that for all $\ell<\ell_{0} \Delta(a, b, \ell)$ is well defined and

$$
\frac{|\Delta(a, b, \ell)|}{\ell}<\frac{r(a, b)}{4}, \forall \ell<\ell_{0} .
$$

The functions being continuous, there exists $\epsilon(a, b)<\ell_{0}$ such that for all $(x, v)$ in $\mathcal{B}(a, \epsilon(a, b)) \times \mathcal{B}(b, \epsilon(a, b))$, we have,

$$
\sigma_{\mathbb{R}^{n}}^{-}(x)<t, \sigma_{\mathbb{R}^{n}}^{-}(x+\ell v)<t, \forall \ell \leq \epsilon(a, b),
$$

and

$$
\left|\frac{\partial h}{\partial x}\left(X\left(x, t_{a, b}\right)\right) \frac{\partial X}{\partial x}\left(x, t_{a, b}\right) v\right| \geq \frac{r(a, b)}{2}, \frac{|\Delta(x, v, \ell)|}{\ell}<\frac{r(a, b)}{3}, \forall \ell \leq \epsilon(a, b) .
$$

The set $\bigcup_{(a, b) \in \mathrm{Cl}\left(\mathcal{A}^{\prime \prime}\right) \times \mathcal{S}_{n}} \mathcal{B}(a, \epsilon(a, b)) \times \mathcal{B}(b, \epsilon(a, b))$ is a covering by open subsets of the compact set $\mathrm{Cl}\left(\mathcal{A}^{\prime \prime}\right) \times \mathcal{S}_{n}$. Hence, we can extract a finite set of $\left(a_{i}, b_{i}\right)_{i=1, \ldots, N}$ in $\operatorname{Cl}\left(\mathcal{A}^{\prime \prime}\right) \times \mathcal{S}_{n}$ such that

$$
\operatorname{Cl}\left(\mathcal{A}^{\prime \prime}\right) \times \mathcal{S}_{n} \subset \bigcup_{\left(a_{i}, b_{i}\right) \in \operatorname{Cl}\left(\mathcal{A}^{\prime \prime}\right) \times \mathcal{S}_{n}} \mathcal{B}\left(a_{i}, \epsilon\left(a_{i}, b_{i}\right)\right) \times \mathcal{B}\left(b_{i}, \epsilon\left(a_{i}, b_{i}\right)\right) .
$$

\footnotetext{
${ }^{10} \mathcal{S}_{n}$ denotes the subset of $\mathbb{R}^{n}$ defined as $\mathcal{S}_{n}=\left\{x \in \mathbb{R}^{n},|x|=1\right\}$
} 
Let $\mathcal{C}_{l}$ be the compact set defined by

$$
\mathcal{C}_{d}=\bigcup_{i=1, \ldots, N} \mathcal{C}_{d, i}, \mathcal{C}_{d, i}=\left\{w \in \mathbb{R}^{n}, w=X(x+\ell v, t), \begin{array}{l}
t \in\left[t_{\left.a_{i}, b_{i}, 0\right]},\right. \\
x \in \mathcal{B}\left(a_{i}, \epsilon\left(a_{i}, b_{i}\right)\right) \\
v \in \mathcal{B}\left(b_{i}, \epsilon\left(a_{i}, b_{i}\right)\right) \\
\ell \in\left[0, \epsilon\left(a_{i}, b_{i}\right)\right]
\end{array}\right\}
$$

The function $(t, x, v, \ell) \mapsto X(x+\ell v, t)$ being continuous and well defined on the set of interest due to (5.13) this set is compact and correctly defined. Finally, if we denote $\epsilon_{m}=\min _{i=1, \ldots, N} \epsilon\left(a_{i}, b_{i}\right)$ we can introduce the compact set

$$
\mathcal{R}=\left\{\left(x_{1}, x_{2}\right) \in \operatorname{Cl}\left(\mathcal{A}^{\prime \prime}\right) \times \operatorname{Cl}\left(\mathcal{A}^{\prime \prime}\right),\left|x_{1}-x_{2}\right| \geq \epsilon_{m}\right\} .
$$

On another hand, consider $c$ and $d$ in $\mathcal{R}$. With Assumption 2, there exists a time $t(c, d)$ in $\left(\max \left\{\sigma_{\mathbb{R}^{n}}^{-}(c), \sigma_{\mathbb{R}^{n}}^{-}(d)\right\}, 0\right]$ such that

$$
|h(X(c, t(c, d)))-h(X(d, t(c, d)))|>0 .
$$

The functions $X$ and $h$ being continuous, there exists $\epsilon(c, d)$ such that for all $\left(x_{1}, x_{2}\right)$ in $\mathcal{B}(c, \epsilon(c, d)) \times \mathcal{B}(d, \epsilon(c, d))$ we have,

$$
\sigma_{\mathbb{R}^{n}}^{-}\left(x_{1}\right)<t(c, d), \sigma_{\mathbb{R}^{n}}^{-}\left(x_{2}\right)<t(c, d),
$$

and,

$$
\left|h\left(X\left(x_{1}, t(c, d)\right)\right)-h\left(X\left(x_{2}, t(c, d)\right)\right)\right|>0 .
$$

The set $\bigcup_{(c, d) \in \mathcal{R}} \mathcal{B}(c, \epsilon(c, d)) \times \mathcal{B}(d, \epsilon(c, d))$ is a covering by open subsets of the compact set $\mathcal{R}$. Hence, we can extract a finite set of $\left(c_{i}, d_{i}\right)$ in $\mathcal{R}$ such that

$$
\mathcal{R} \subset \bigcup_{\left(c_{i}, d_{i}\right) \in \mathcal{R}} \mathcal{B}\left(c_{i}, \epsilon\left(c_{i}, d_{i}\right)\right) \times \mathcal{B}\left(d_{i}, \epsilon\left(c_{i}, d_{i}\right)\right) .
$$

Let now, $\mathcal{C}_{l}$ be the compact set defined by

$$
\mathcal{C}_{r}=\bigcup_{i} \mathcal{C}_{r, i}, \mathcal{C}_{r, i}=\left\{w \in \mathbb{R}^{n}, w=X(x, t), \begin{array}{l}
t \in\left[t_{c_{i}, d_{i}}, 0\right] \\
x \in \mathcal{B}\left(c_{i}, \epsilon\left(c_{i}, d_{i}\right)\right) \cup \mathcal{B}\left(d_{i}, \epsilon\left(c_{i}, d_{i}\right)\right)
\end{array}\right\} .
$$

Finally, consider the set $\mathcal{A}^{\prime}$ be any bounded open set containing $\mathcal{C}_{r} \cup \mathcal{C}_{d}$. Then, we have the required distinguishability property. Indeed, consider $x_{1}$ and $x_{2}$ in $\operatorname{Cl}\left(\mathcal{A}^{\prime \prime}\right)$ such that $x_{1} \neq x_{2}$. Two cases may be distinguished.

1. If $\left|x_{1}-x_{2}\right| \leq \epsilon_{m}$. With $v=\frac{x_{1}-x_{2}}{\left|x_{1}-x_{2}\right|}$, there exists $i$ such that, $\left(x_{1}, v\right)$ is in $\mathcal{B}\left(a_{i}, \epsilon\left(a_{i}, b_{i}\right)\right) \times \mathcal{B}\left(b_{i}, \epsilon\left(a_{i}, b_{i}\right)\right)$. Moreover, we have $\left|x_{1}-x_{2}\right| \leq \epsilon_{m} \leq \epsilon\left(a_{i}, b_{i}\right)$. Hence, with 5.13), we have,

$$
\sigma_{\mathbb{R}^{n}}^{-}\left(x_{1}\right)<t\left(a_{i}, b_{i}\right), \sigma_{\mathbb{R}^{n}}^{-}\left(x_{2}\right)<t\left(a_{i}, b_{i}\right),
$$

and moreover, with 5.15 , it yields,

$$
\left.X\left(x_{1}, t\right) \in \mathcal{C}_{d, i}, X\left(x_{2}, t\right) \in \mathcal{C}_{d, i}, \forall t \in\left[t_{(} a_{i}, b_{i}\right), 0\right] .
$$

Hence, with the definition of $\mathcal{A}^{\prime}$, it yields,

$$
\sigma_{\mathcal{A}^{\prime}}^{-}\left(x_{1}\right)<t\left(a_{i}, b_{i}\right), \sigma_{\mathcal{A}^{\prime}}^{-}\left(x_{2}\right)<t\left(a_{i}, b_{i}\right) .
$$


On another hand, if we denote $\ell=\left|x_{1}-x_{2}\right|$ we have,

$$
\begin{aligned}
& h\left(X\left(x_{1}, t\left(a_{i}, b_{i}\right)\right)\right)-h\left(X\left(x_{2}, t\left(a_{i}, b_{i}\right)\right)\right)=\ell \frac{\partial h}{\partial x}\left(X\left(x_{1}, t_{a, b}\right)\right) \frac{\partial X}{\partial x}\left(x_{1}, t_{a, b}\right) v \\
&+\Delta\left(x_{1}, v, \ell\right) .
\end{aligned}
$$

which gives,

$$
\begin{aligned}
\left|h\left(X\left(x_{1}, t\left(a_{i}, b_{i}\right)\right)\right)-h\left(X\left(x_{2}, t\left(a_{i}, b_{i}\right)\right)\right)\right| & \geq \\
\ell & {\left[\frac{\partial h}{\partial x}\left(X\left(x_{1}, t_{a, b}\right)\right) \frac{\partial X}{\partial x}\left(x_{1}, t_{a, b}\right) v-\frac{\left|\Delta\left(x_{1}, v, \ell\right)\right|}{\ell}\right] . }
\end{aligned}
$$

Hence, with (5.14) it yields,

$$
\left|h\left(X\left(x_{1}, t\left(a_{i}, b_{i}\right)\right)\right)-h\left(X\left(x_{2}, t\left(a_{i}, b_{i}\right)\right)\right)\right| \geq \ell \frac{r(a, b)}{6}>0 .
$$

2. If $\left|x_{1}-x_{2}\right| \geq \epsilon_{m}$. In this case, $\left(x_{1}, x_{2}\right)$ is in $\mathcal{R}$. Hence, there exists $i$ such that $\left(x_{1}, x_{2}\right)$ is in $\mathcal{B}\left(c_{i}, \epsilon\left(c_{i}, d_{i}\right)\right) \times \mathcal{B}\left(d_{i}, \epsilon\left(c_{i}, d_{i}\right)\right)$. Hence, with (5.16), we have,

$$
\sigma_{\mathbb{R}^{n}}^{-}\left(x_{1}\right)<t\left(c_{i}, d_{i}\right), \sigma_{\mathbb{R}^{n}}^{-}\left(x_{2}\right)<t\left(c_{i}, d_{i}\right),
$$

and moreover, with 5.18, it yields,

$$
\left.X\left(x_{1}, t\right) \in \mathcal{C}_{r, i}, X\left(x_{2}, t\right) \in \mathcal{C}_{r, i}, \forall t \in\left[t_{(} c_{i}, d_{i}\right), 0\right] .
$$

Hence, with the definition of $\mathcal{A}^{\prime}$, it yields,

$$
\sigma_{\mathcal{A}^{\prime}}^{-}\left(x_{1}\right)<t\left(c_{i}, d_{i}\right), \sigma_{\mathcal{A}^{\prime}}^{-}\left(x_{2}\right)<t\left(c_{i}, d_{i}\right) .
$$

On another hand, with 5.17) it yields,

$$
\left|h\left(X\left(x_{1}, t\left(c_{i}, d_{i}\right)\right)\right)-h\left(X\left(x_{2}, t\left(c_{i}, d_{i}\right)\right)\right)\right|>0 .
$$

Hence, in both cases, the points $x_{1}$ and $x_{2}$ are distinguished before leaving $\mathcal{A}^{\prime}$ which proves the first point of the Proposition.

Now, let $(x, v)$ be in $\operatorname{Cl}\left(\mathcal{A}^{\prime \prime}\right) \times \mathbb{R}^{n}$ with $v \neq 0$. Note that $\left(x, \frac{v}{|v|}\right)$ is in $\operatorname{Cl}\left(\mathcal{A}^{\prime \prime}\right) \times \mathcal{S}_{n}$. Hence there exists $i$ such that

$$
\sigma_{\mathcal{A}^{\prime}}^{-}(x)<t\left(a_{i}, b_{i}\right)
$$

and,

$$
\frac{\partial h}{\partial x}\left(X\left(x, t\left(a_{i}, b_{i}\right)\right)\right) \frac{\partial X}{\partial x}\left(x, t\left(a_{i}, b_{i}\right)\right) \frac{v}{|v|} \neq 0 .
$$

This gives the second point of the Proposition.

5.5. Proof of Proposition 3.6. For a given $\lambda$, we denote $T_{\lambda}: \mathbb{R}^{n} \rightarrow \mathbb{C}$ the function (when it exists) defined by

$$
T_{\lambda}(x)=\int_{-\infty}^{0} \exp (-\lambda s) h(\breve{X}(x, s)) d s .
$$

Following the proof of Proposition 3.3 , there exists a negative real number $\rho$ such that the function $T_{\lambda}(x)$ is $C^{2}$ provided $\operatorname{Re}(\lambda)<\rho$. Moreover, with equation 5.11 in the proof of Lemma 3.3 , it yields for all $\lambda$ in $\mathbb{C}$ with $\operatorname{Re}(\lambda)<\rho$

$$
\int_{-\infty}^{0} \exp (-2 \operatorname{Re}(\lambda) s)\left|\frac{\partial h(\breve{X}(x, s))}{\partial x} v\right|^{2} d s<+\infty .
$$


The rest of the proof of this proposition follows the same line as the one of Proposition 3.5 (a proof of which is given in [3]) and is based on the use of Coron's Lemma.

LEMma 5.1 (Coron [7]). Let $\Gamma$ and $\Upsilon$ be open subsets of $\mathbb{C}$ and $\mathbb{R}^{2 n}$ respectively. Let $g: \Upsilon \times \Gamma \rightarrow \mathbb{C}^{p}$ be a function which is holomorphic in $\lambda$ for each $x$ in $\Upsilon$ and $C^{1}$ in $x$ for each $\lambda$ in $\Gamma$. If, for each pair $(x, \lambda)$ in $\Upsilon \times \Gamma$ for which $g(x, \lambda)$ is zero it is possible to find, for at least one of the $p$ components $g_{j}$ of $g$, an integer $k$ satisfying:

$$
\begin{aligned}
& \frac{\partial^{i} g_{j}}{\partial \lambda^{i}}(x, \lambda)=0 \forall i \in\{0, \ldots, k-1\}, \\
& \frac{\partial^{k} g_{j}}{\partial \lambda^{k}}(x, \lambda) \neq 0,
\end{aligned}
$$

then the following set has zero Lebesgue measure in $\mathbb{C}^{n+1}$ :

$$
\mathcal{I}=\bigcup_{x \in \Upsilon}\left\{\left(\lambda_{1}, \ldots, \lambda_{n+1}\right) \in \Gamma^{n+1} g\left(x, \lambda_{1}\right)=\ldots=g\left(x, \lambda_{n+1}\right)=0\right\}
$$

This result has been established by Coron in [7, Lemma 3.2] in a stronger form except for the very minor point that, here, $g$ is not $C^{\infty}$ in both $x$ and $\lambda$. A proof of this specific result can be found in 3 .

Let $\Gamma$ and $\Upsilon$ be open sets defined by:

$$
\Gamma=\{\lambda \in \mathbb{C}, \operatorname{Re}(\lambda)<\rho\}, \Upsilon=\left\{w=(x, v) \in \mathcal{A}^{\prime \prime} \times \mathbb{R}^{n}: v \neq 0\right\} .
$$

Now, consider the function $\mathcal{G} T: \Upsilon \times \Gamma \rightarrow \mathbb{C}$ defined by :

$$
\mathcal{G} T(w, \lambda)=\frac{\partial T_{\lambda}}{\partial x}(x) v
$$

with $w=(x, v)$. This function is $C^{1}$ in $w$ in $\Upsilon$ for all $\lambda$ in $\Gamma$. Moreover, it can be shown in 26, chap 19, p. 367] that the Theorem of Morera and Fubini yields that this function is holomorphic in $\lambda$ in $\Gamma$, for all $w$ in $\Upsilon$. Consequently, with (5.20), Plancherel Theorem can be employed to get for all $w$ in $\Upsilon$,

$$
\frac{1}{2 \pi} \int_{-\infty}^{+\infty}|\mathcal{G} T(w, \operatorname{Re}(\lambda)+i s)|^{2} d s=\int_{-\infty}^{0} \exp (-2 \operatorname{Re}(\lambda) s)\left|\frac{\partial h(\breve{X}(x, s))}{\partial x} v\right|^{2} d s .
$$

Now, for all $w$ in $\Upsilon$, exploiting inequality (3.9) and the continuity with respect to the time, it yields the existence of an open interval $\left(t_{0}, t_{1}\right)$ with $\sigma_{\mathcal{A}^{\prime}}^{-}(x)<t_{0}$ for which

$$
\left|\frac{\partial h(X(x, s))}{\partial x} v\right|>0 \quad \forall s \in\left(t_{0}, t_{1}\right) .
$$

With the definition of the modified system (3.6), it yields

$$
h(\breve{X}(x, s))=h(X(x, s)) \quad \forall s \in\left(t_{0}, t_{1}\right) .
$$

Hence, with (5.25), the last equality and inequality (5.26) yield that:

$$
\int_{-\infty}^{+\infty}|\mathcal{G} T(w, \operatorname{Re}(\lambda)+i s)|^{2} d s>0 .
$$


This implies that for all $w$ in $\Upsilon$, the function $\lambda \mapsto \mathcal{G} T(w, \lambda)$ is not identically zero on $\Gamma$. Since this function is holomorphic, it yields that for all $(w, \lambda)$ in $\Upsilon \times \Gamma$, there exists, for at least one of the $n+1$ components $\mathcal{G} T_{j}$ of $\mathcal{G} T$, an integer $k$ which satisfies:

$$
\left\{\begin{array}{l}
\frac{\partial^{i} \mathcal{G} T_{j}}{\partial \lambda^{i}}(w, \lambda)=0 \quad \forall i \in\{0, \ldots, k-1\}, \\
\frac{\partial^{k} \mathcal{G} T_{j}}{\partial \lambda^{k}}(w, \lambda) \neq 0 .
\end{array}\right.
$$

Hence, employing Coron's Lemma with $\mathcal{G}$ as the $g$ function, and by using (5.24), it allows to conclude that the set $\mathcal{I}_{l e}$ defined by :

$$
\mathcal{I}_{l e}=\left\{\left(\lambda_{1}, \ldots, \lambda_{n+1}\right) \in \Gamma^{n+1}: \exists(x, v) \in \Upsilon: \frac{\partial T_{\lambda_{i}}}{\partial x}(x) v=0 \forall i \in\{1, \ldots, n+1\}\right\}
$$

has a zero Lebesgue measure in $\mathbb{C}^{n+1}$.

5.6. Proof of Proposition 4.2. This result has been obtained in [1]. We consider the following sequence of sets $V_{m} \subset \mathbb{R}^{n} \times \mathbb{R}^{n}$ defined as,

$$
V_{\ell}=\left\{\left(x_{1}, x_{2}\right) \in \mathbb{R}^{n} \times \mathbb{R}^{n} \mid H_{\ell}\left(x_{1}\right)=H_{\ell}\left(x_{2}\right)\right\} .
$$

By definition of $H_{\ell}$, we have $V_{k+1} \subseteq V_{k}$. Hence, the sequence $V_{k}$ is a decreasing sequence of analytic subsets of $\mathbb{R}^{n} \times \mathbb{R}^{n}$.

The ideal of the ring of analytic functions being Notherian, by [20, corollary 1, p.99], $V_{\ell}$ is a stationary sequence. Hence, there exists $k$, such that

$$
V_{\ell} \cap(\mathrm{Cl}(\mathcal{A}) \times \mathrm{Cl}(\mathcal{A}))=V_{k} \cap(\mathrm{Cl}(\mathcal{A}) \times \mathrm{Cl}(\mathcal{A})), \forall k>\ell .
$$

This shows that the system (2.1) is differentially distinguishable of order $k$ in $\mathrm{Cl}(\mathcal{A})$. Indeed, assume there exists $x_{1}$ and $x_{2}$ in $\mathrm{Cl}(\mathcal{A}) \times \mathrm{Cl}(\mathcal{A})$ such that $H_{k}\left(x_{1}\right)=$ $H_{k}\left(x_{2}\right)$. Hence, it yields that $\left(x_{1}, x_{2}\right)$ is in $V_{k}$ and by stationarity $\left(x_{1}, x_{2}\right)$ is also in $V_{\ell}$ for all $k \geq \ell$. This implies that,

$$
L_{f}^{k} h\left(x_{1}\right)=L_{f}^{k} h\left(x_{2}\right), \forall k .
$$

On the other hand, the functions describing the model being analytic, we know that for all $x$ in $\mathrm{Cl}(\mathcal{A})$ the output function

$$
t \mapsto h(X(x, t))
$$

is an analytic function of the time with infinite convergence radius. Consequently, for all $x$ in $\mathrm{Cl}(\mathcal{A})$, there exists a positive real number $t_{x}<\sigma_{\mathbb{R}^{n}}^{+}(x)$ such that

$$
h(X(x, t))=h(x)+\sum_{j=1}^{+\infty} L_{f}^{j-1} h(x) \frac{t^{j}}{j !}, \forall 0 \leq t<t_{x} .
$$

Hence, $P_{\mathbb{R}^{n}}\left(x_{1}\right)_{[0, \sigma)}=P_{\mathbb{R}^{n}}\left(x_{2}\right)_{[0, \sigma)}$ where $\sigma=\max \left\{\sigma_{\mathbb{R}^{n}}^{+}\left(x_{1}\right), \sigma_{\mathbb{R}^{n}}^{+}\left(x_{2}\right)\right\}$ and consequently $x_{1}=x_{2}$ due to the fact that the system is distinguishable at $\mathcal{O}$ in $\mathbb{R}^{n}$. 
5.7. Proof of Proposition 4.3. We consider the following sequence of sets $V_{\ell} \subset \mathbb{R}^{n} \times \mathbb{R}^{n}$ with $\ell$ in $\mathbb{N}$ defined as,

$$
V_{\ell}=\left\{(x, v) \in \mathbb{R}^{n} \times \mathbb{R}^{n} \mid \frac{\partial H_{\ell}}{\partial x}(x) v=0\right\} .
$$

By definition of $H_{\ell}$, we have $V_{k+1} \subseteq V_{k}$. Hence, the sequence $V_{k}$ is a decreasing sequence of analytic subsets of $\mathbb{R}^{n} \times \mathbb{R}^{n}$.

The ideal of the ring of analytic functions being Notherian, by [20, corollary 1 , p.99], $V_{\ell}$ is a stationary sequence in all compact subset. Hence, there exists $k$, such that

$$
\begin{aligned}
& V_{\ell} \cap\left\{(x, v) \in \mathbb{R}^{n} \times \mathbb{R}^{n}|x \in \mathrm{Cl}(\mathcal{A}),| v \mid \leq 1\right\}= \\
& V_{k} \cap\left\{(x, v) \in \mathbb{R}^{n} \times \mathbb{R}^{n}|x \in \mathrm{Cl}(\mathcal{A}),| v \mid \leq 1\right\}, \forall k>\ell .
\end{aligned}
$$

This shows that the system (2.1) satisfies the differential rank condition of order $k$ in $\mathcal{O}$. Indeed, assume there exists $(x, v)$ in $\mathcal{A} \times \mathbb{R}^{n}$ such that $\frac{\partial H_{k}}{\partial x}(x) v=0$. Hence, it yields that $\left(x, \frac{v}{|v|}\right)$ is in $V_{k}$ and by stationarity $\left(x, \frac{v}{|v|}\right)$ is also in $V_{\ell}$ for all $\ell \geq k$. This implies that,

$$
\frac{\partial L_{f} h^{\ell-1}}{\partial x}(x) \frac{v}{|v|}=\frac{\partial L_{f} h^{\ell-1}}{\partial x}(x) v=0, \forall \ell .
$$

On the other hand, the functions describing the model being analytic with infinite convergence radius, the output function

$$
(t \mapsto h(X(x, t)),
$$

is an analytic function of the time with infinite convergence radius. Consequently, for all $x$ in $\operatorname{Cl}(\mathcal{A})$ it yields,

$$
h(X(x, t))=h(x)+\sum_{j=1}^{+\infty} L_{f}^{j-1} h(x) \frac{t^{j}}{j !}, \forall 0 \leq t \leq \sigma_{\mathbb{R}^{n}}^{+}(x) .
$$

Consequently, it yields,

$$
\frac{\partial h(X(x, t))}{\partial x} v=\frac{\partial h}{\partial x}(x) v+\sum_{j=1}^{\infty} \frac{\partial L_{f} h^{j-1}}{\partial x}(x) v \frac{t^{j}}{j !}=0, \forall t .
$$

The system being infinitesimally observable in $\mathcal{O}$, it yields that $v=0$. Hence, the system satisfies the differential rank condition of order $k$ in $\mathrm{Cl}(\mathcal{A})$.

5.8. Proof of Theorem 4.1. As done in [3], the idea is to consider a family of $m \times m$ Hurwitz matrices $k A$ where $k$ is a positive real number which will be selected sufficiently large and $A=\operatorname{Diag}\left\{\lambda_{1}, \ldots, \lambda_{m}\right\}$. Given a bounded open set $\mathcal{A}$, a corresponding solution to the partial differential equation 3.1 is given as

$$
T_{k}(x)=\int_{-\infty}^{0} \exp (-k A s) B_{1 m} h(\breve{X}(x, s)) d s
$$

where $\breve{X}: \mathbb{R}^{n} \times \mathbb{R} \rightarrow \mathbb{R}^{n}$ is the solution of the modified system (3.6)-(3.7) where $\delta_{b}$ is any positive real number and $\mathcal{A}^{\prime}$ is a bounded open set such that $\operatorname{Cl}(\mathcal{A}) \subset$ 
$\mathcal{A}^{\prime}$. As shown in Proposition 3.3 there exists a negative real number $\rho$ such that if $\max _{i=1, \ldots, n+1}\left\{\operatorname{Re}\left(\lambda_{i}\right)\right\}<\rho$ then for all $k \geq 1$, the function $T_{k}$ given in (5.27) is well defined, $C^{2}$ and a solution to the partial differential equation (3.1).

By integrating by part it yields that the function $T_{k}$ satisfies for all $x$ in $\mathbb{R}^{n}$

$$
T_{k}(x)=-(k A)^{-1} B_{1 m} h(x)+(k A)^{-1} \int_{-\infty}^{0} \exp (-k A s) B_{1 m} L_{\breve{f}} h(\breve{X}(x, s)) d s,
$$

which is well defined due to the fact that the set $\mathcal{A}^{\prime}+\delta_{b}$ is bounded and backward invariant along the trajectories of the system (3.6). And with successive integration by part, we obtain

$$
T_{k}(x)=(k A)^{-m} R_{k}(x)-K^{-1} S H_{m}(x),
$$

with the notations :

$$
S=\left(\begin{array}{ccc}
\lambda_{1}^{-1} & \ldots & \lambda_{1}^{-m} \\
\vdots & \vdots & \vdots \\
\lambda_{m}^{-1} & \ldots & \lambda_{m}^{-m}
\end{array}\right) \quad, \quad K=\operatorname{Diag}\left(k, \ldots, k^{m}\right),
$$

and $R_{k}: \mathbb{R}^{n} \rightarrow \mathbb{C}^{m}$ is the function defined by

$$
R_{k}(x)=\int_{-\infty}^{0} \exp (-s k A) B L_{\breve{f}}^{m} h(\breve{X}(x, s)) d s .
$$

Again, this function is well defined since the function $x \mapsto L_{\breve{f}}^{m} h(\breve{X}(x, s))$ is uniformly bounded in $s$ for all $x$. Moreover, with Proposition 3.3 , this function is $C^{1}$ for $k$ sufficiently large. More precisely, we have for all $x$ in $\operatorname{Cl}(\mathcal{A})$

$$
\left|\frac{\partial R_{k}}{\partial x}(x)\right| \leq \int_{-\infty}^{0}\left|\exp (-s k A) B_{1 m} \frac{\partial L_{\breve{f}}^{m} h}{\partial x}(\breve{X}(x, s)) \frac{\partial \breve{X}}{\partial x}(x, s)\right| d s,
$$

and consequently, with 5.10 , it yields

$$
\left|\frac{\partial R_{k}}{\partial x}(x)\right| \leq \int_{-\infty}^{0} \exp \left(s\left[\rho_{1}-k \max _{i}\left\{\operatorname{Re}\left\{\lambda_{i}\right\}\right]\right)\left|\frac{\partial L_{\breve{f}}^{m} h}{\partial x}(\breve{X}(x, s)) \sqrt{n}\right| d s .\right.
$$

Consequently we obtain the following inequality.

$$
\left|R_{k}\left(x_{1}\right)-R_{k}\left(x_{2}\right)\right| \leq \frac{c_{0}}{\rho_{1}-k \rho}\left|x_{1}-x_{2}\right|, \forall\left(x_{1}, x_{2}\right) \in \mathrm{Cl}(\mathcal{A})^{2},
$$

where $c_{0}$ is the positive real number defined as,

$$
c_{0}=\max _{x \in \mathrm{Cl}\left(\mathcal{A}^{\prime}+\delta_{b}\right)}\left|\frac{\partial L_{\breve{f}}^{m} h}{\partial x}(x)\right| .
$$

Hence, for all $\left(x_{1}, x_{2}\right)$ in $\mathrm{Cl}(\mathcal{A})^{2}$ it gives,

$$
\begin{aligned}
\left|T_{k}\left(x_{1}\right)-T_{k}\left(x_{2}\right)\right| & \geq\left|K^{-1} S\left(H_{m}\left(x_{1}\right)-H_{m}\left(x_{2}\right)\right)\right|-\left|(k A)^{-m}\left(R_{k}\left(x_{1}\right)-R_{k}\left(x_{2}\right)\right)\right| \\
& \geq \frac{1}{|K|\left|S^{-1}\right|}\left|H_{m}\left(x_{1}\right)-H_{m}\left(x_{2}\right)\right|-\frac{|k \rho|^{-m} c_{0}}{\rho_{1}-k \rho}\left|x_{1}-x_{2}\right|, \\
& \geq k^{-m}\left[\frac{1}{\left|S^{-1}\right|}\left|H_{m}\left(x_{1}\right)-H_{m}\left(x_{2}\right)\right|-\frac{|\rho|^{-m} c_{0}}{\rho_{1}-k \rho}\left|x_{1}-x_{2}\right|\right] .
\end{aligned}
$$


On another hand, with Lemma 3.2 Assumption 4 and 5 , it yields that there exists a positive real number $L_{H}$ such that for all $\left(x_{1}, x_{2}\right)$ in $\operatorname{Cl}(\mathcal{A})^{2}$ we have,

$$
\left|H_{m}\left(x_{1}\right)-H_{m}\left(x_{2}\right)\right| \geq L_{H}\left|x_{1}-x_{2}\right| .
$$

Hence, it yields,

$$
\left|T_{k}\left(x_{1}\right)-T_{k}\left(x_{2}\right)\right| \geq k^{-m}\left[\frac{L_{H}}{\left|S^{-1}\right|}-\frac{|\rho|^{-m} c_{0}}{\rho_{1}-k \rho}\right]\left|x_{1}-x_{2}\right| .
$$

Note that for all $k$ sufficiently large, and with Proposition 3.1 this implies that there exists a continuous function $T_{k}^{*}: \mathbb{C}^{m} \rightarrow \mathbb{R}^{n}$ such that for all $(x, z)$ in $\mathcal{A} \times \mathbb{C}^{m}$ inequality 4.3 is obtained with

$$
M_{k}(z)=\sqrt{n} k^{-m}\left[\frac{L_{H}}{\left|S^{-1}\right|}-\frac{|\rho|^{-m} c_{0}}{\rho_{1}-k \rho}\right]\left[|z|+\max _{x \in \operatorname{C1}(\mathcal{A})}\left|T_{k}(x)\right|\right] .
$$

Note moreover that the set $\mathcal{A}^{\prime}+\delta_{b}$ being backward invariant along the trajectory of the system (3.6), it yields

$$
\left|T_{k}(x)\right| \leq \frac{c_{1}}{k \rho}, \forall x \in \mathrm{Cl}(\mathcal{A}),
$$

where $c_{1}$ is the positive real number defined as,

$$
c_{1}=\max _{x \in \mathrm{C} 1\left(\mathcal{A}^{\prime}+\delta_{b}\right)}|h(x)| .
$$

Note that there exists $k^{*}$ such that for all $k \geq k^{*}$ we have

$$
\sqrt{n} k^{-m}\left[\frac{1}{\left|S^{-1}\right|} L_{H}-\frac{|\rho|^{-m} c_{0}}{\rho_{1}-k \rho}\right] c_{1} \exp \left(k \rho t_{f}\right) \leq \epsilon .
$$

Hence we get the result.

6. Conclusion. In this paper is presented a sufficient condition guaranteeing that a nonlinear Luenberger observer as introduced in [27, [12] and [3] converges exponentially towards the state of the model. Moreover, it is shown that in the complete observability concept this observer is tunable. These facts may be used to design some output feedback based on this observer. For instance some of these arguments have been used in output regulations in [10].

7. acknowledgement. The author thanks Laurent Praly for many fruitful discussions.

\section{REFERENCES}

[1] V. Andrieu. Bouclage de sortie et observateur. Ecole des Mines de Paris, 2005.

[2] V. Andrieu. Exponential convergence of nonlinear Luenberger observers. In Proc. 49st IEEE Conference on Decision and Control, 2010.

[3] V. Andrieu and L. Praly. On the existence of Kazantzis-Kravaris / Luenberger Observers. SIAM Journal on Control and Optimization, 45(2):432-456, 2006.

[4] V. Andrieu and L. Praly. A unifying point of view on output feedback designs for global asymptotic stabilization. Automatica, 45:1789-1798, 2009.

[5] G. Besançon. Nonlinear observers and applications, volume 363. Springer Verlag, 2007. 
[6] D. Carnevale and A. Astolfi. Hybrid observer for global frequency estimation of saturated signals. IEEE Trans. Automatic Control, 54(10):2461-2464, 2009.

[7] J.-M. Coron. On the stabilization of controllable and observable systems by an output feedback law. Mathematics of Control, Signals, and Systems, 7(3):187-216, 1994.

[8] J. P. Gauthier, H. Hammouri, and I. Kupka. Observers for nonlinear systems. In 30th IEEE Conference on Decision and Control, volume 2, 1991.

[9] J.-P. Gauthier and I. Kupka. Deterministic observation theory and applications. Cambridge University Press, 2001.

[10] A. Isidori, L. Praly, and L. Marconi. About the existence of locally lipschitz output feedback stabilizers for nonlinear systems. SIAM J. Control Optim., 48(5):3389-3402, 2010.

[11] P. Jouan and J.P. Gauthier. Finite singularities of nonlinear systems. output stabilization, observability, and observers. Journal of Dynamical and Control systems, 2(2):255-288, 1996.

[12] N. Kazantzis and C. Kravaris. Nonlinear observer design using Lyapunov's auxiliary theorem. Systems 83 Control Letters, 34:241-247, 1998.

[13] G. Kreisselmeier and R. Engel. Nonlinear observers for autonomous Lipschitz continuous systems. IEEE Transactions on Automatic Control, 48(3), 2003.

[14] A.J. Krener and A. Isidori. Linearization by output injection and nonlinear observers. Systems E Control Letters, 3(1):47-52, 1983.

[15] A.J. Krener and W. Respondek. Nonlinear observers with linearizable error dynamics. SIAM Journal on Control and Optimization, 23(2):197-216, 1985.

[16] A.J. Krener and M. Xiao. Nonlinear observer design in the siegel domain,. SIAM Journal on Control and Optimization, 41(3):932-953, 2002.

[17] D. Luenberger. Observing the state of a linear system. IEEE Transactions on Military Electronics, MIL-8:74-80, 1964.

[18] L. Marconi and L. Praly. Uniform practical nonlinear output regulation. IEEE Transactions on Automatic Control, 53(5):1184-1202, 2008.

[19] E.J. McShane. Extension of range of functions. Bull. Amer. Math. Soc, 40(12):837-842, 1934.

[20] R. Narasimhan. Introduction to the theory of analytic spaces, volume 25. Springer-Verlag, 1966.

[21] F. Poulain, L. Praly, and R. Ortega. An Observer for Permanent Magnet Synchronous Motors with Currents and Voltages as only Measurements. In Proc. 47th IEEE Conference on Decision and Control, pages 5390-5395, 2008.

[22] L. Praly. Fonctions de Lyapunov, Stabilité et Stabilisation. Ecole Nationale Supérieure des Mines de Paris, 2008.

[23] L. Praly, A. Isidori, and L. Marconi. A new observer for an unknown harmonic oscillator. In 17th International Symposium on Mathematical Theory of Networks and Systems, pages 24-28, 2006.

[24] A. Rapaport and A. Maloum. Design of exponential observers for nonlinear systems by embedding. International Journal of Robust and Nonlinear Control, 14(3):273-288, 2004.

[25] W. Respondek, A. Pogromsky, and H. Nijmeijer. Time scaling for observer design with linearizable error dynamics. Automatica, 40(2):277-285, 2004.

[26] W. Rudin. Real and complex analysis. McGraw-Hill, Inc., 1966.

[27] A.N. Shoshitaishvili. Singularities for projections of integral manifolds with applications to control and observation problems. Theory of singularities and its applications, 1:295, 1990.

[28] A. Teel and L. Praly. Global stabilizability and observability imply semi-global stabilizability by output feedback. Systems \& Control Letters, 22(5):313-325, 1994. 